\title{
THE THREE-POINT FUNCTION OF GENERAL PLANAR MAPS
}

\author{
ÉRIC FUSY AND EMMANUEL GUITTER
}

\begin{abstract}
We compute the distance-dependent three-point function of general planar maps and of bipartite planar maps, i.e., the generating function of these maps with three marked vertices at prescribed pairwise distances. Explicit expressions are given for maps counted by their number of edges only, or by both their numbers of edges and faces. A few limiting cases and applications are discussed.
\end{abstract}

\section{INTRODUCTION}

The statistics of distances in planar maps is a subject of constant interest, whose study already produced a lot of remarkable results but still leaves many open questions. A key ingredient in this study was the discovery by Schaeffer [15, 11] (giving a reformulation of a bijection due to Cori and Vauquelin [12]) of a distance-preserving bijective coding of planar quadrangulations (later generalized to planar maps with arbitrary face degrees [6]) by decorated trees, reducing de facto a number of distance statistics problems to the simpler question of enumerating these trees. This approach was used to obtain the so-called twopoint function which, by enumerating maps with two marked vertices at a prescribed distance, provides a measure of the statistics of distances between two random points in the map. In a first stage, the explicit expression of the two-point function was derived in [5, 10] for a number of families of maps with controlled bounded face degrees (triangulations, quadrangulations, ...), corroborating the original prediction of Ambjørn and Watabiki 3. The restriction to maps with bounded face degrees was lifted in a second, very recent, stage thanks to the discovery by Ambjørn and Budd [2] of yet another distance-preserving bijective coding of planar quadrangulations, now by general planar maps with unbounded face degrees. This latter coding, combined with the original Schaeffer bijection, allowed in turn to get an explicit expression for the two-point function of general planar maps controlled by their number of edges only or by both their numbers of edges and faces. This approach was then generalized in 7. to obtain the two-point function of other families of maps and hypermaps with unbounded face degrees, controlled by their number of edges or their numbers of hyperedges and faces.

The more involved question of the three-point function, now enumerating maps with three marked vertices at prescribed pairwise distances, hence providing a refined information on the correlations between mutual distances, was so far solved only in the simplest case of planar quadrangulations [8. The solution relies on a natural extension by Miermont 14] of the original Schaeffer bijection and constituted so far the most advanced result on the statistics of distances within maps at the discrete level.

In this paper, we show how to take advantage of the new Ambjørn-Budd bijection to get an explicit expression for the three-point function of general planar maps controlled by both their numbers of edges and faces. We also obtain the three-point function of the subclass of general planar maps made of bipartite planar maps.

The paper is organized as follows: Section 2 explains how to use the Ambjørn-Budd bijection to obtain a bijective coding of bi-pointed and tri-pointed planar maps, i.e., maps with respectively two and three marked vertices, which preserves the information on the distance between the marked vertices. The coding involves what we call $(s, t)$ - and $(s, t, u)$-welllabelled maps, which are maps with respectively two and three faces, whose vertices carry labels subject to a number of constraints involving the parameters $s, t$, and $u$, themselves 
directly related to the prescribed pairwise distances between the marked vertices. The case of bipartite planar maps is obtained by keeping only a subclass of these well-labelled maps, which we call very-well-labelled. We then exploit this coding in Section 3 to derive explicit expressions for the two-point (Sect. 3.1) and three-point (Sect. 3.2) functions of general planar maps controlled by their number of edges only. The case of bipartite planar maps is addressed in Sect. 3.3. Our derivation relies on the preliminary knowledge of the generating functions of a number of well-labelled or very-well-labelled objects, an information which follows either from previously known expressions or from the resolution of new recursion relations. We then repeat our analysis in Section 4 to obtain bivariate two- and three-point functions, with a control on both the number of edges and the number of faces, in the case of general maps (Sect. 4.1) or of bipartite maps (Sect. 4.2). We also discuss at this level the limit of tri-pointed maps having a minimal number of faces (in practice one or two faces). Section 5 is devoted to a number of applications of our results in the scaling limit of maps with a large number of edges (and a fixed weight per face). This includes the average number of vertices or edges lying on a geodesic path between two given far away vertices. We gather our conclusions in Section 6.

\section{BiJective CODING OF BI-POINTED AND TRI-POINTED MAPS}

2.1. Definitions. A map denotes a connected graph embedded on the sphere. A bipartite map is a map with all faces of even degree, and a quadrangulation is a map with all faces of degree 4. A well-labelled map (resp. very-well-labelled map) is a map $M$ together with an assignment: $\ell: V \rightarrow \mathbf{Z}$ of integers to its vertices, such that for each edge $e=\{u, v\}$ of $M$, $|\ell(v)-\ell(u)| \leq 1$ (resp. $|\ell(v)-\ell(u)|=1$ ). A very-well-labelled map is necessarily bipartite. A vertex of a well-labelled map is called a local max (resp. local min) if it is not smaller (resp. not larger) than any of its neighbours. For a face $f$ of a well-labelled map, denote by $\min (f)$ (resp. $\max (f))$ the minimum (resp. maximum) label over vertices incident to $f$. Denote by $\widehat{\mathcal{M}}$ the set of well-labelled maps and by $\widehat{\mathcal{Q}}$ the set of very-well-labelled quadrangulations.

2.2. The Ambjørn-Budd bijection. Let $\Phi$ be the mapping from $\widehat{\mathcal{Q}}$ to $\widehat{\mathcal{M}}$ such that, for $q \in \widehat{\mathcal{Q}}, \Phi(q)$ is the map obtained from $q$ by applying the local rules of Figure 1 (left-part) to each face of $q$, then deleting all local max and all edges of $q$. Similarly let $\Phi^{-}$be the mapping from $\widehat{\mathcal{Q}}$ to $\widehat{\mathcal{M}}$ such that, for $q \in \widehat{\mathcal{Q}}, \Phi^{-}(q)$ is the map obtained from $q$ by applying the local rules of Figure 1 (right-part) to each face of $q$, then deleting all local mins and all edges of $q$. Ambjørn and Budd showed 2 that $\Phi$ and $\Phi^{-}$are bijections between $\widehat{\mathcal{Q}}$ and $\widehat{\mathcal{M}}$ that preserve several parameters, from which it follows (see Figure 2 for an example):

Theorem 1 (Ambjørn-Budd). The mapping $\Lambda=\Phi^{-} \circ \Phi^{-1}$ is a bijection between $\widehat{\mathcal{M}}$ and $\widehat{\mathcal{M}}$ that preserves the number of edges, such that for $M \in \widehat{\mathcal{M}}$ and $M^{\prime}=\Lambda(M)$, each local min $v$ of $M$ corresponds to a face $f^{\prime}$ of $M^{\prime}$, with $\ell(v)=\min \left(f^{\prime}\right)-1$, and each face $f$ of $M$ corresponds to a local max $v^{\prime}$ of $M^{\prime}$, with $\max (f)+1=\ell\left(v^{\prime}\right)$.

Remark 1. Note that each edge $e^{\prime}$ of labels $i-i$ in $M^{\prime}=\Lambda(M)$ is "dual" to an edge of labels $(i-1)-(i-1)$ in $M$, such that (when $M$ and $M^{\prime}$ are superimposed), $e^{\prime}$ crosses $e$ (within the same face of the associated $q \in \widehat{\mathcal{Q}}$ ). This also ensures that $\Lambda$ and $\Lambda^{-1}$ preserve the property of being very-well-labelled.

We now state a useful lemma (in view of proving bijections for bi-pointed and tri-pointed maps later on).

Lemma 1. Let $M \in \widehat{\mathcal{M}}$ and $M^{\prime}=\Lambda(M)$, considered as superimposed (via the associated $q \in \widehat{\mathcal{Q}})$. Consider two edges $e_{1}, e_{2}$ of $M^{\prime}$ incident to a vertex $u$ of $M^{\prime}$, of label $i$, such that the extremities $v$ and $w$ of $e_{1}$ and $e_{2}$ have label $i+1$. Let $S$ be the clockwise angular sector between $e_{1}$ and $e_{2}$ around $u$. Then there is an edge of $M$ leaving $u$ in the sector $S$ and ending at a vertex of label $i-1$. 

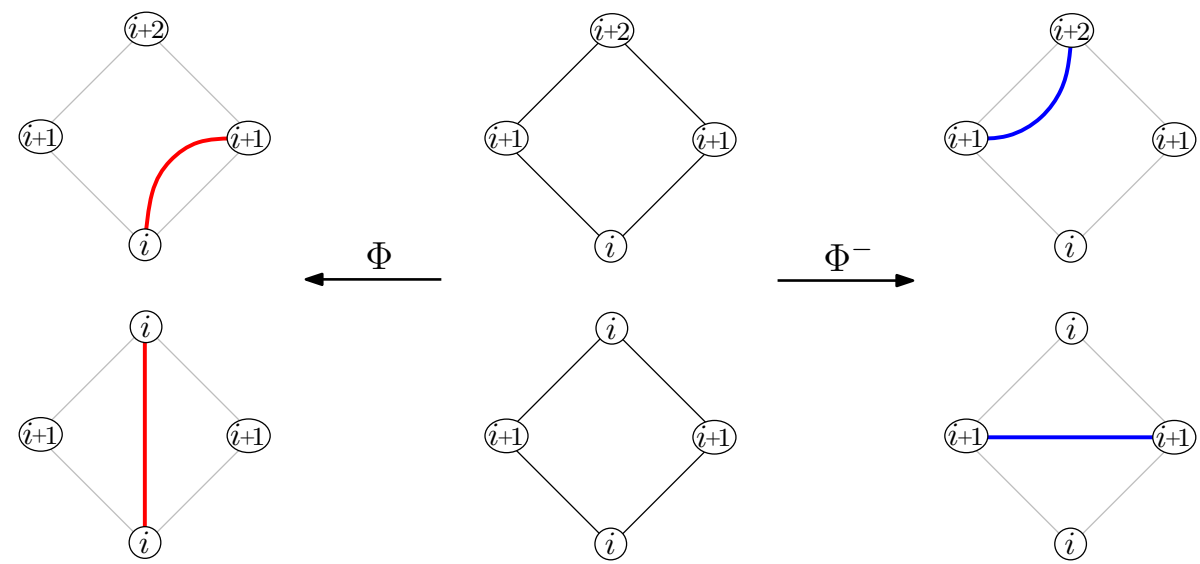

(i)

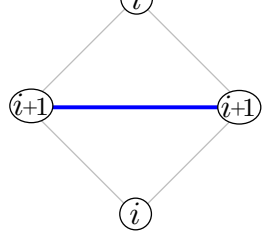

Figure 1. Local rules of the bijections $\Phi$ (left) and $\Phi^{-}$(right) applied within each face of a very-well-labelled quadrangulation. The rules on the right are those of the Schaeffer bijection [15, 11.

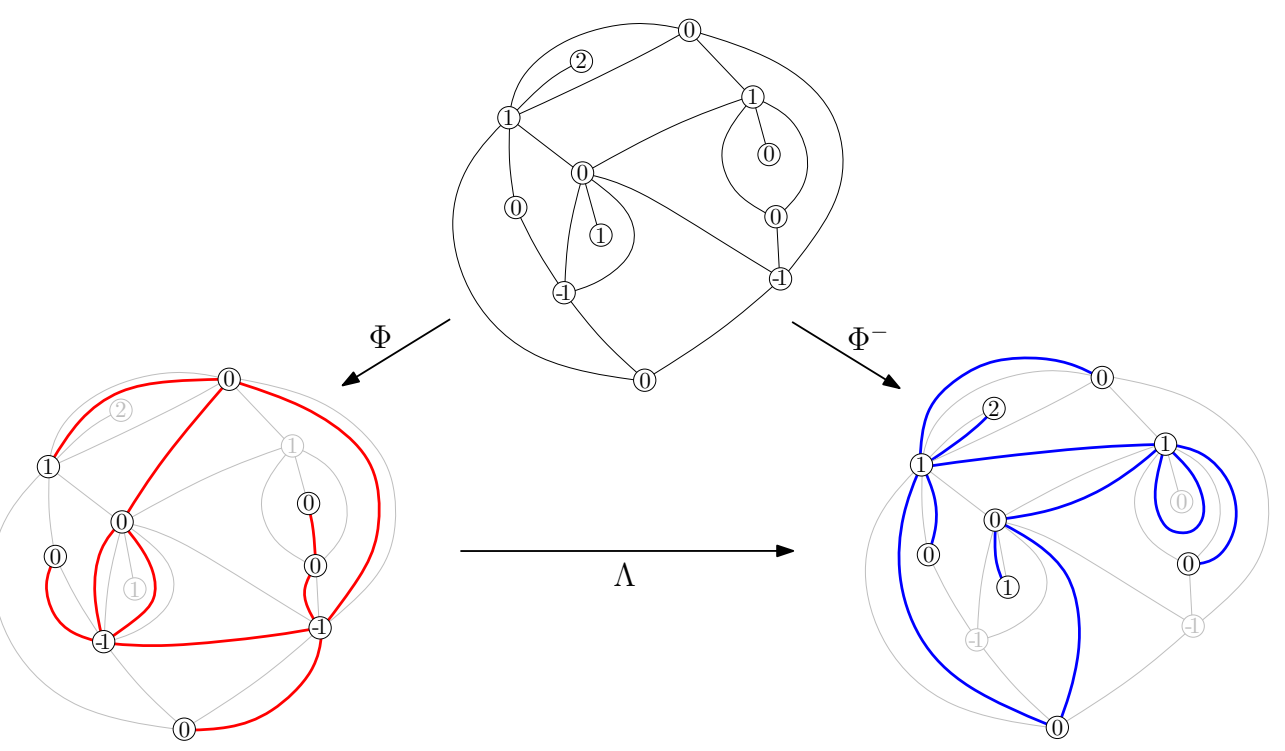

Figure 2. The Ambjørn-Budd bijection on an example: each face $f^{\prime}$ of $M^{\prime}=\Lambda(M)$ corresponds to a local min $v$ of $M$ (with $\ell(v)=\min \left(f^{\prime}\right)-1$, and with $v$ within $f^{\prime}$ when $M$ and $M^{\prime}$ are superimposed), and each face $f$ of $M$ corresponds to a local max $v^{\prime}$ of $M^{\prime}$ (with $\ell\left(v^{\prime}\right)=\max (f)+1$, and with $v^{\prime}$ within $f$ when $M$ and $M^{\prime}$ are superimposed).

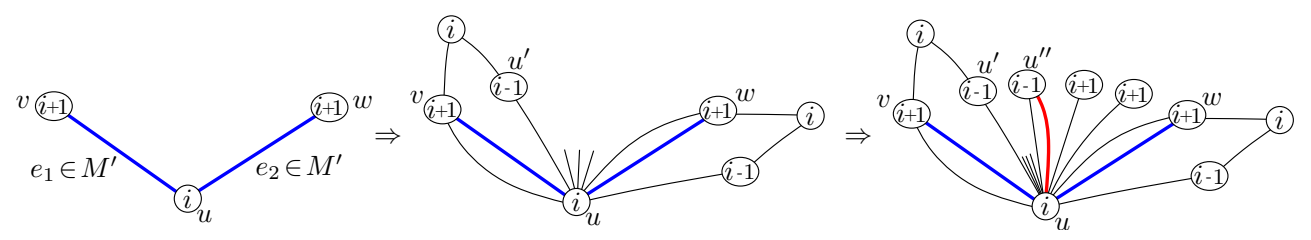

Figure 3. The situation in the proof of Lemma 1 
Proof. The situation is shown in Figure 3 . Let $q$ be the very-well-labelled quadrangulation associated to $M$ (via $\Phi$ ) and to $M^{\prime}$ (via $\Phi^{-}$). The local rules of $\Phi^{-}$ensure that $q$ has an edge from $u$ to $v$ (just to the left of $e_{1}$ ), an edge from $u$ to $w$ (just to the left of $e_{2}$ ), and that the next edge of $q$ after $e_{1}$ in clockwise order around $u$ leads to a vertex of label $i-1$; denote by $u^{\prime}$ this vertex. Starting from $u^{\prime}$, let $u^{\prime \prime}$ be the last neighbour of $u$ of label $i-1$ (in clockwise order around $u$ ) before $w$. Then the local rules of $\Phi$ ensure that there is an edge of $M$ from $u$ to $u^{\prime \prime}$, and this edge is in $S$.

2.3. Bijection for bi-pointed maps. A bi-pointed map is a map with two (different and distinguished) marked vertices, denoted $v_{1}$ and $v_{2}$. The distance between $v_{1}$ and $v_{2}$ is called the two-point distance of $M$ and is denoted $d_{12}(M)$. For $s, t$ two positive integers, define an $[s, t]$-well-labelled map as a bi-pointed well-labelled map such that $v_{1}$ and $v_{2}$ are the only local min, and have respective labels $-s$ and $-t$. Define an $(s, t)$-well-labelled map as a well-labelled map with exactly two faces (which are distinguished) $f_{1}$ and $f_{2}$, such that $\min \left(f_{1}\right)=-s+1$ and $\min \left(f_{2}\right)=-t+1$. In such a well-labelled map, a border-vertex (resp. border-edge) is a vertex (resp. an edge) incident to $f_{1}$ and $f_{2}$. An $(s, t$ )-well-labelled map is said to be of type $A$ if the minimum label over all border-vertices is 0 , and none of the borderedges has labels $0-0$. An $(s, t)$-well-labelled map is said to be of type $B$ if the minimum label over all border-vertices is 0 , and there is at least one border-edge with labels $0-0$.

Proposition 1. For $s, t \geq 1$, the mapping $\Lambda$ induces a bijection between $[s, t]$-well-labelled maps and $(s, t)$-well-labelled maps. For $M$ an $[s, t]$-well-labelled map and $M^{\prime}=\Lambda(M)$, one has $d_{12}(M)=s+t$ iff $M^{\prime}$ is of type $A$, and $d_{12}(M)=s+t-1$ iff $M^{\prime}$ is of type $B$.

Proof. We just have to show the statements of the second sentence (the first sentence immediately follows from Theorem 1). Let $\Gamma$ be the submap of $M^{\prime}$ formed by the border-vertices and border-edges (note that $\Gamma$ is a cycle). Denote by $a$ the minimum label over all vertices of $\Gamma$. Let $P$ be a path in $M$ from $v_{1}$ to $v_{2}$, and let $p$ be the first intersection of $P$ with $\Gamma$. Two cases can occur: if $p$ is at a vertex of $\Gamma$, of label $i$, then the portion of $P$ before $p$ (resp. after $p$ ) has length at least $s+i$ (resp. at least $t+i$ ) because the label-increment along each edge is at most 1 ; hence $P$ has length at least $s+t+2 i$. If $p$ is at the middle of an edge $e^{\prime}$ of $\Gamma$, of labels $i-i$, then $e^{\prime}$ is "dual" to an edge $e$ of $M$ of labels $(i-1)-(i-1)$, and $P$ hits $p$ when traversing $e$. Again, the portion of $P$ before $e$ (resp. after $e$ ) has length at least $s+i-1$ (resp. at least $t+i-1$ ), so that $P$ has length at least $s+t+2 i-1$. This observation ensures that if $d_{12}(M)=s+t$ then the labels $i$ on $\Gamma$ cannot all be positive, hence $a \leq 0$, and if $d_{12}(M)=s+t-1$ then there exists on $\Gamma$ a vertex with label $i<0$ or an edge with labels $0-0$, hence either we have $a<0$ or we have $a=0$ and there is at least one border-edge of labels $0-0$.

We now aim at obtaining an upper bound for $d_{12}(M)$ in terms of $a$. Observe that two cases can arise: (i) no border-edge has labels $a-a$, (ii) at least one border-edge has labels $a-a$. In case (i), let $v$ be a border-vertex of label $a$. Its neighbours on $\Gamma$ have label $a+1$, so by Lemma 1, $v$ has (in $M$ ) a neighbour $w$ of label $a-1$ that belongs to $f_{1}$ (note that $w$ is strictly in $f_{1}$ since all border-vertices have label at least $a$ ). Let $v_{1}$ be the local min of $M$ in $f_{1}$. If $w \neq v_{1}$, then $w$ is not a local min of $M$, so that $w$ has (in $M$ ) a neighbour of label $a-2$, strictly in $f_{1}$. Continuing this way one builds a label-decreasing path (staying strictly in $f_{1}$ ) from $v$ to $v_{1}$, hence of length $s+a$. Similarly one can build a label-decreasing path from $v$ to $v_{2}$, of length $t+a$. Hence $d_{12}(M) \leq s+t+2 a$. In case (ii), let $e^{\prime}$ be a border-edge of labels $a-a$, and let $e \in M$ be its dual edge, with labels $(a-1)-(a-1)$. Let $w_{1}$ be the extremity of $e$ in $f_{1}$ and $w_{2}$ the extremity of $e$ in $f_{2}$ (note that $w_{1}$ is strictly in $f_{1}$ and $w_{2}$ is strictly in $f_{2}$ ). Again, if $w_{1} \neq v_{1}$, then $w_{1}$ has (in $M$ ) a neighbour of label $a-2$, and continuing this way one builds a label-decreasing path $P_{1}$ between $w_{1}$ and $v_{1}$, of length $s+a-1$. Similarly one can build a label-decreasing path $P_{2}$ between $w_{2}$ and $v_{2}$, of length $t+a-1$. Concatenating $P_{1}, e$, and $P_{2}$ yields a path between $v_{1}$ and $v_{2}$ of length $s+t+2 a-1$. Hence $d_{12}(M) \leq s+t+2 a-1$. Using the first paragraph of the proof, we 

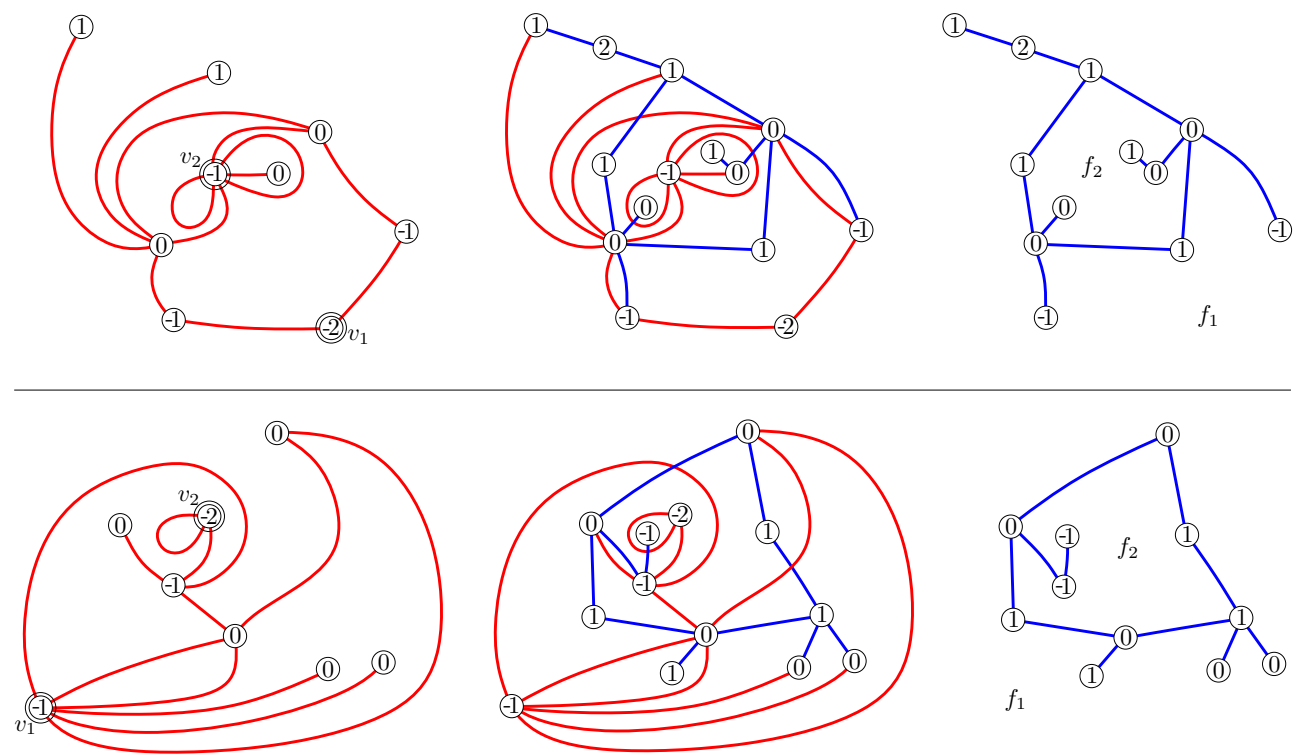

Figure 4. Top: the left-part shows a bi-pointed map of two-point distance 3 , endowed with its [1,2]-well-labelling; the right-part shows the associated (1,2)-well-labelled map of type A (and the middle-part shows both drawings superimposed). Bottom: the left-part shows a bi-pointed map of two-point distance 2, endowed with its [1,2]-well-labelling; the right-part shows the associated $(1,2)$-well-labelled map of type B (and the middle-part shows both drawings superimposed).

conclude that, if $d_{12}(M)=s+t$, then $a=0$ and we are in case (i), i.e., $M^{\prime}$ is of type A; and if $d_{12}(M)=s+t-1$, then $a=0$ and we are in case (ii), i.e., $M^{\prime}$ is of type B.

Conversely, if $M^{\prime}$ is of type $\mathrm{A}$, then by the arguments of the first paragraph, $d_{12}(M) \geq s+t$; and by the arguments of the second paragraph, for each border-vertex $v$ of label 0 one can build a path of length $s+t$ from $v_{1}$ to $v_{2}$ passing by $v$ at distance $s$ from $v_{1}$. Hence $d_{12}(M)=s+t$. And similarly if $M^{\prime}$ is of type $\mathrm{B}$, then by the arguments of the first paragraph, $d_{12}(M) \geq s+t-1$; and by the arguments of the second paragraph, for each border-edge $e^{\prime}$ of labels $0-0$ one can build a path of length $s+t-1$ from $v_{1}$ to $v_{2}$, with $e$ as the $s$ th edge along the path. Hence $d_{12}(M)=s+t-1$.

The proposition then yields the following bijection (see Figure 4 for examples):

Theorem 2. Let $s, t$ be two positive integers.

$(A)$ : the mapping $\Lambda$ induces a bijection (via $[s, t]$-well-labelled maps) between bi-pointed maps of two-point distance $s+t$, and $(s, t)$-well-labelled maps of type A; the bi-pointed map is bipartite iff the $(s, t)$-well-labelled map of type $A$ is very-well-labelled 1 .

(B): the mapping $\Lambda$ induces a bijection (via $[s, t]$-well-labelled maps) between bi-pointed maps of two-point distance $s+t-1$, and $(s, t)$-well-labelled maps of type $B$.

In both bijections $(A)$ and $(B)$, each face of the bi-pointed map corresponds to a local max in the associated $(s, t)$-well-labelled map.

Proof. It suffices to show that a bi-pointed map $M$ of two-point distance $s+t$ (resp $s+t-1)$ can be uniquely labelled as an $[s, t]$-well-labelled map, and then to apply Proposition 1 . Consider the labelling-assignment $\ell_{0}(v)=\min \left(d\left(v, v_{1}\right)-s, d\left(v, v_{2}\right)-t\right)$, where $d(u, w)$ denotes

\footnotetext{
${ }^{1}$ Note that an $(s, t)$-well-labelled map which is very-well-labelled (for short an $(s, t)$-very-well-labelled map) is of type A iff the minimum label over border vertices is 0 .
} 
the distance (in $M$ ) between $u$ and $w$. Clearly $\ell_{0}$ is a well-labelling and any vertex $v \notin\left\{v_{1}, v_{2}\right\}$ is not a local min. And since $|s-t|<s+t=d\left(v_{1}, v_{2}\right)$ (recall that $s$ and $t$ are positive), it is easy to see that $v_{1}$ and $v_{2}$ are local min, of respective labels $-s$ and $-t$. This proves the existence of such a labelling. To prove uniqueness, consider a labelling $\ell$ that makes $M(s, t)$-well-labelled. For any vertex $v$ of $M$ which is not a local min, one can find a label-decreasing path $P$ to a local min, say $v_{1}$. Then $\ell(v)-\ell\left(v_{1}\right)=|P| \geq d_{12}(M)$, hence $\ell(v) \geq \ell_{0}(v)$. Moreover, since the increments along edges are at most 1 , one also has (considering increments along the geodesic paths from $v$ to $v_{1}$ and from $v$ to $\left.v_{2}\right) \ell(v) \leq \ell_{0}(v)$. Hence $\ell(v)=\ell_{0}(v)$ and we have the uniqueness of such a labelling.

Finally note that, for a bi-pointed map $M$ with $d_{12}(M)=s+t, \ell_{0}$ is a very-well labelling iff $M$ is bipartite (indeed, if $M$ is bipartite, for $v$ a vertex of $M$, either both $d\left(v, v_{1}\right)-s$ and $d\left(v, v_{2}\right)-t$ are even, or both $d\left(v, v_{1}\right)-s$ and $d\left(v, v_{2}\right)-t$ are odd, depending on whether $v$ is in one vertex-color or the other).

Remark 2. The arguments of the proof of Proposition 1 imply that $-M$ denoting the bipointed map and $M^{\prime}$ the corresponding $(s, t)$-well-labelled map — in case (A) of Theorem 2 each border-vertex of label 0 in $M^{\prime}$ corresponds to a vertex $v$ of $M$ that belongs to a (not necessarily unique) geodesic path from $v_{1}$ to $v_{2}$, with $v$ at distance $s$ from $v_{1}$, and in case (B) of Theorem 2, each border-edge of labels $0-0$ in $M^{\prime}$ corresponds to an edge $e$ of $M$ that belongs to a (not necessarily unique) geodesic path from $v_{1}$ to $v_{2}$, with $e$ the $s$ th edge along the path.

2.4. Bijection for tri-pointed maps. A tri-pointed map is a map $M$ with three (different and distinguished) marked vertices, denoted $v_{1}, v_{2}, v_{3}$. Denote by $d_{12}, d_{13}, d_{23}$ the distances between the marked vertices, i.e., $d_{i j}$ is the distance between $v_{i}$ and $v_{j}$.

For $s, t, u$ three positive integers, define an $[s, t, u]$-well-labelled map as a tri-pointed welllabelled map such that $v_{1}, v_{2}, v_{3}$ are the only local min, and have respective labels $-s,-t,-u$. Define an $(s, t, u)$-well-labelled map as a well-labelled map with exactly three faces (which are distinguished) $f_{1}, f_{2}, f_{3}$, such that $\min \left(f_{1}\right)=-s+1, \min \left(f_{2}\right)=-t+1$, and $\min \left(f_{3}\right)=-u+1$. For such a map, a border-vertex is a vertex incident to at least two different faces, and a border-edge is an edge incident to two different faces. More precisely, for $1 \leq i<j \leq 3$, an $(i, j)$-border vertex is a vertex incident to both $f_{i}$ and $f_{j}$ (and possibly also to the other face), and an $(i, j)$-border edge is an edge incident to both $f_{i}$ and $f_{j}$. An $(s, t, u)$-well-labelled map is said to be of type $A$ if the minimum label over all border-vertices is 0 , there is no border-edge of labels $0-0$, and for all $1 \leq i<j \leq 3$ there is at least one $(i, j)$-border vertex of label 0 . An $(s, t, u)$-well-labelled map is said to be of type $B$ if the minimum label over all border-vertices is 0 , and for all $1 \leq i<j \leq 3$ there is at least one $(i, j)$-border edge of labels $0-0$. Note in particular that, in an $(s, t, u)$-well-labelled map of type A or B, any of the three faces is adjacent to the two others.

Proposition 2. For $s, t, u$ three positive integers, the mapping $\Lambda$ induces a bijection between $[s, t, u]$-well-labelled maps and $(s, t, u)$-well-labelled maps. For $M$ an $[s, t, u]$-well-labelled map and $M^{\prime}=\Lambda(M)$, the distances $d_{12}, d_{13}, d_{23}$ of $M$ satisfy

$$
d_{12}=s+t, \quad d_{13}=s+u, \quad d_{23}=t+u
$$

iff $M^{\prime}$ is of type $A$, and satisfy

$$
d_{12}=s+t-1, \quad d_{13}=s+u-1, \quad d_{23}=t+u-1
$$

iff $M^{\prime}$ is of type $B$.

Proof. Again we just have to show the statements of the second sentence (the first sentence immediately follows from Theorem 1). Let $\Gamma$ be the embedded subgraph of $M^{\prime}$ formed by the border-vertices and border-edges ( $\Gamma$ cuts the sphere into 3 components, and at this point we can not yet exclude the possibility that $\Gamma$ consists of two disjoint cycles). For $1 \leq i<j \leq 3$, let $\Gamma_{i j}$ be the subgraph of $\Gamma$ made of vertices and edges incident to $f_{i}$ and $f_{j}$. Let $a$ be the 
minimum label over all vertices of $\Gamma$. By similar arguments as in the first paragraph of the proof of Proposition 1 in case $d_{12}=s+t, d_{13}=s+u, d_{23}=t+u$ we must have $a \leq 0$, and in case $d_{12}=s+t-1, d_{13}=s+u-1, d_{23}=t+u-1$ we must either have $a<0$ or have $a=0$ and there is an edge in $\Gamma$ of labels $0-0$.

Next we prove an upper bound for the two-point distance between two of the marked vertices in terms of $a$. Two cases can arise: (i) no border edge has labels $a-a$, (ii) at least one border-edge has labels $a-a$. In case (i) let $v$ be a border-vertex of label $a$, say without loss of generality that $v$ is incident to $f_{1}$ and $f_{2}$. By the same arguments (using Lemma 1) as in the proof of Proposition 1, there is a label-decreasing path in $f_{1}$ from $v$ to $v_{1}$, and there is a label-decreasing path in $f_{2}$ from $v$ to $v_{2}$, so that the concatenation of these two paths yields a path of length $s+t+2 a$ between $v_{1}$ and $v_{2}$. In case (ii) let $e^{\prime}$ be a border-edge of labels $a-a$, and let $e$ be the edge of labels $(a-1)-(a-1)$ in $M$ that is dual to $e^{\prime}$. Say without loss of generality that $e^{\prime}$ is incident to $f_{1}$ and $f_{2}$. Again by the same arguments (using Lemma 1 ) as in the proof of Proposition 1. there is a path of length $s+t+2 a-1$ (passing by $e$ ) between $v_{1}$ and $v_{2}$. Hence, together with the first paragraph, we conclude that, if the distances satisfy $d_{12}=s+t, d_{13}=s+u, d_{23}=t+u$, then we must have $a=0$ and there is no border-edge of labels $0-0$, and if the distances satisfy $d_{12}=s+t-1, d_{13}=s+u-1, d_{23}=t+u-1$, then we must have $a=0$ and there is a border-edge of labels $0-0$.

Assume $d_{12}=s+t, d_{13}=s+u, d_{23}=t+u$. We now prove that, for any $1 \leq i<j \leq 3$, $\Gamma_{i j}$ contains a vertex of label 0 . Take (without loss of generality) $i=1$ and $j=2$, and consider a path $P$ of length $s+t$ between $v_{1}$ and $v_{2}$. Assume for contradiction that $P$ does not meet $\Gamma_{12}$. Let $p$ be the first point of intersection of $P$ with $\Gamma$ and let $p^{\prime}$ be the last point of intersection of $P$ with $\Gamma$. Since $P$ does not meet $\Gamma_{12}, p$ is in $\Gamma_{13}$ and not incident to $f_{2}$, and $p^{\prime}$ is in $\Gamma_{23}$ and not incident to $f_{1}$; in particular $p \neq p^{\prime}$. Note that $p$ is either at a vertex of label $b$ with $b \geq 0$ or at the middle of an edge of $\Gamma_{13}$ of labels $b-b$ with $b \geq 1$; and similarly $p^{\prime}$ is either at a vertex of label $c$ with $c \geq 0$ or at the middle of an edge of $\Gamma_{23}$ of labels $c-c$ with $c \geq 1$. In all four cases it is easily checked that the length of $P$ is strictly larger than $s+t$, which yields a contradiction. Hence $P$ has to cross $\Gamma_{12}$, either at a vertex of label $b \geq 0$, or at an edge of labels $b-b$ with $b \geq 1$. In the second case, $P$ has length at least $s+t+2 b-1>s+t$, which is impossible, and in the first case $P$ has length at least $s+t+2 b$, so that $b=0$. We conclude that $\Gamma_{12}$ (and more generally any $\Gamma_{i j}$ ) has a vertex of label 0 . (Note that, in particular, all $\Gamma_{i j}$ are non empty, which excludes the possibility that $\Gamma$ is made of two disjoint cycles, hence $\Gamma$ is connected.) We have thus shown that, if $d_{12}=s+t, d_{13}=s+u, d_{23}=t+u$, then $M^{\prime}$ is of type A. Similarly, if $d_{12}=s+t-1, d_{13}=s+u-1, d_{23}=t+u-1$, one can prove that, for any $1 \leq i<j \leq 3$, $\Gamma_{i j}$ contains an edge of labels $0-0$ (again by considering a geodesic path from $v_{i}$ to $v_{j}$, then showing that $P$ must have length larger than $s+t-1$ in all cases, except for the case where $P$ crosses $\Gamma_{i j}$ at an edge of labels $\left.0-0\right)$. Hence $M^{\prime}$ is of type B.

Conversely, if $M^{\prime}$ is of type $\mathrm{A}$, then one can check at first that the distances satisfy $d_{12} \geq s+t, d_{13} \geq s+u, d_{23} \geq t+u$ (by the arguments in the first paragraph of the proof of Proposition 1). Then one can also check that $d_{12} \leq s+t, d_{13} \leq s+u, d_{23} \leq t+u$ (for $d_{12}$, take a vertex of label 0 on $\Gamma_{12}$, and construct a label-decreasing path from $v$ to $v_{1}$, of length $s$, and a label-decreasing path from $v$ to $v_{2}$, of length $t$ ). Hence if $M^{\prime}$ is of type $\mathrm{A}$, then the distances satisfy $d_{12}=s+t, d_{13}=s+u, d_{23}=t+u$. Similarly, if $M^{\prime}$ is of type $\mathrm{B}$, then the distances satisfy $d_{12}=s+t-1, d_{13}=s+u-1, d_{23}=t+u-1$.

The proposition then yields the following bijection (see Figure 5 for examples):

Theorem 3. Let $d_{12}, d_{13}, d_{23}$ be a triple of positive integers satisfying triangular inequalities.

(A): If $d_{12}+d_{13}+d_{23}$ is even and $d_{12}, d_{13}, d_{23}$ satisfy strict triangular inequalities, let $s, t, u$ be the unique triple of positive integers ${ }^{2}$ such that

$$
d_{12}=s+t, \quad d_{13}=s+u, \quad d_{23}=t+u .
$$

\footnotetext{
${ }^{2}$ given by $s=\left(d_{12}+d_{13}-d_{23}\right) / 2, t=\left(d_{12}+d_{23}-d_{13}\right) / 2, u=\left(d_{13}+d_{23}-d_{12}\right) / 2$.
} 

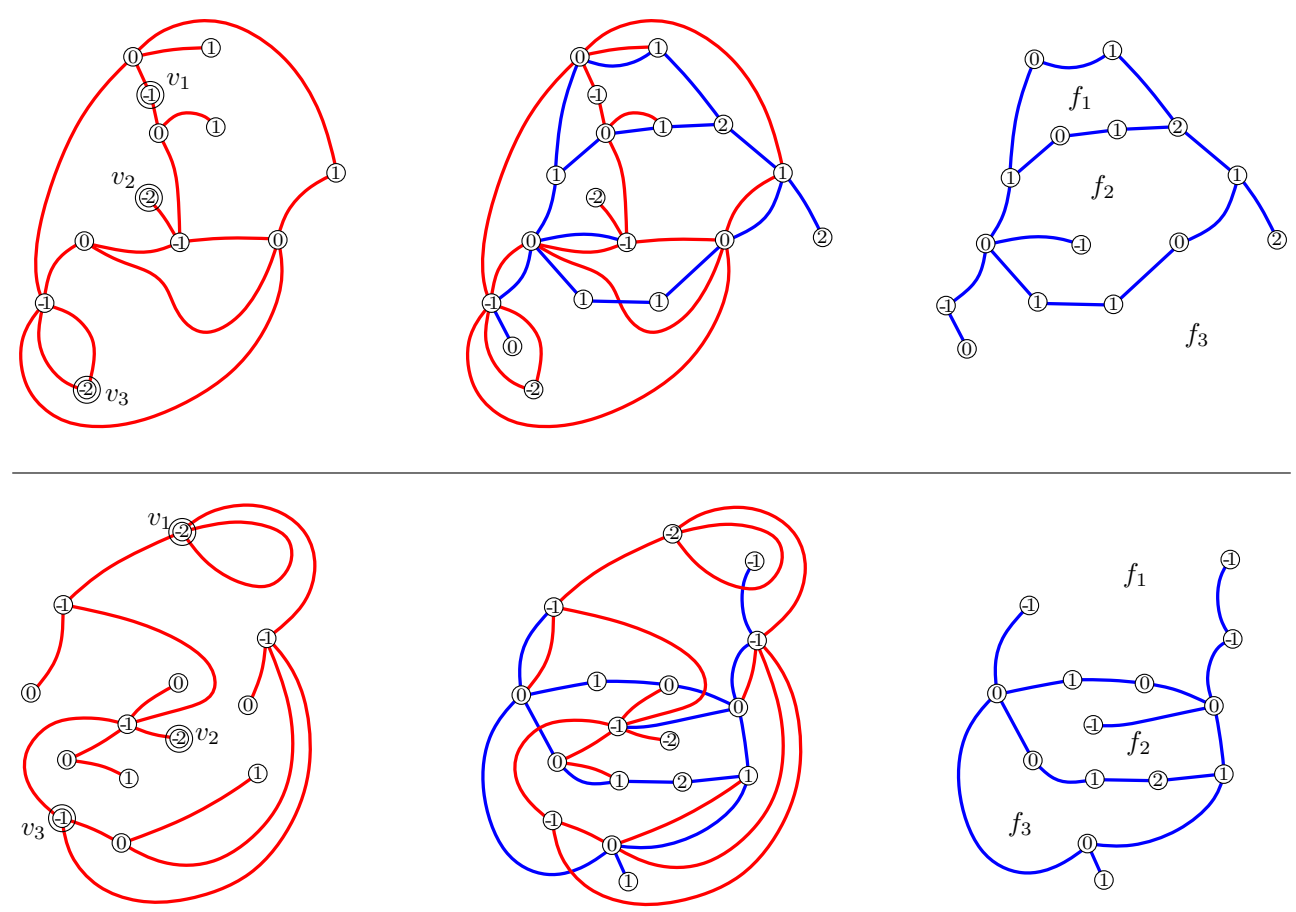

Figure 5. Top: the left-part shows a tri-pointed map of distances $d_{12}=$ $3, d_{13}=3, d_{23}=4$, endowed with its $[1,2,2]$-well-labelling; the right-part shows the associated $(1,2,2)$-well-labelled map of type A (and the middlepart shows both drawings superimposed). Bottom: the left-part shows a tripointed map of distances $d_{12}=3, d_{13}=2, d_{23}=2$, endowed with its [2, 2, 1]well-labelling; the right-part shows the associated $(2,2,1)$-well-labelled map of type B (and the middle-part shows both drawings superimposed).

Then tri-pointed maps with distances $d_{12}, d_{13}, d_{23}$ are in bijection (via $[s, t, u]$-well-labelled maps) with $(s, t, u)$-well-labelled maps of type $A$. The tri-pointed map is bipartite iff the corresponding $(s, t, u)$-well-labelled map of type $A$ is very-well-labelled.

(B): If $d_{12}+d_{13}+d_{23}$ is odd, let $s, t, u$ be the unique triple of positive integers ${ }^{3}$ such that:

$$
d_{12}=s+t-1, \quad d_{13}=s+u-1, \quad d_{23}=t+u-1 .
$$

Then tri-pointed maps with distances $d_{12}, d_{13}, d_{23}$ are in bijection (via $[s, t, u]$-well-labelled maps) with $(s, t, u)$-well-labelled maps of type $B$.

In both bijections $(A)$ and $(B)$, each face of the tri-pointed map corresponds to a local max in the associated $(s, t, u)$-well-labelled map.

Proof. It suffices to show that a tri-pointed map $M$ with distances $s+t, s+u, t+u$ (resp. $s+t-1, s+u-1, t+u-1)$ can be uniquely labelled as an $[s, t, u]$-well-labelled map, and then to apply Proposition 2 . Consider the labelling-assignment $\ell_{0}(v)=\min \left(d\left(v, v_{1}\right)-s, d\left(v, v_{2}\right)-\right.$ $\left.t, d\left(v, v_{3}\right)-u\right)$, where $d(v, w)$ denotes the distance (in $\left.M\right)$ between $v$ and $w$. Clearly $\ell_{0}$ is a well-labelling. Similarly as in the proof of Theorem 2 it can be checked that $\ell_{0}$ is indeed the unique $[s, t, u]$-well-labelling of $M$.

Moreover, for a tri-pointed map $M$ with distances $s+t, s+u, t+u, \ell_{0}$ is a very-well labelling iff $M$ is bipartite (indeed, if $M$ is bipartite, for $v$ a vertex of $M$, either $d\left(v, v_{1}\right)-$ $s, d\left(v, v_{2}\right)-t, d\left(v, v_{3}\right)-u$ are all even, or $d\left(v, v_{1}\right)-s, d\left(v, v_{2}\right)-t, d\left(v, v_{3}\right)-u$ are all odd, depending on whether $v$ is in one vertex-color or the other).

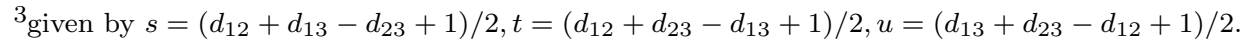


Remark 3. The case where the triangular inequalities on $d_{12}, d_{13}, d_{23}$ are not strict is not covered by Theorem 3 but by Remark 2 after Theorem 2 Indeed, let again $s, t, u$ be the values such that $d_{12}=s+t, d_{13}=s+u, d_{23}=t+u$. When the triangular inequalities are not strict, exactly one of $s, t, u$ is zero (note that at most one of $s, t, u$ can be zero for the three marked vertices to be distinct), say $u=0$. In that case, $v_{3}$ is a vertex on a geodesic path between $v_{1}$ and $v_{2}$, at distance $s$ from $v_{1}$. By Remark 2, tri-pointed maps with distances $d_{12}=s+t, d_{13}=s, d_{23}=t$ are in bijection with $(s, t)$-well-labelled maps of type A with a marked border-vertex $v_{3}$ of label 0 .

\section{TWO- AND THREE-POINT FUNCTIONS}

We shall now make use of Theorems 2 and 3 to derive explicit expressions for the twoand three-point functions of general and bipartite planar maps. Recall that, by two-point (resp. three-point) function, we mean, in all generality, the generating function of bi-pointed (resp. tri-pointed) maps with a prescribed two-point distance $d_{12}$ (resp. with prescribed pairwise distances $d_{12}, d_{13}$ and $d_{23}$ ). We shall first concentrate on the two- and three-point functions of general planar maps, enumerated with a weight $g$ per edge, and then consider the subclass of bipartite planar maps. Note that bi-pointed maps may have a $k$-fold symmetry by "rotation" around their two marked vertices: as customary, these $k$-fold symmetric maps receive an extra conventional weight $1 / k$ (only with this convention is the two-point function simple). In the following, we shall denote by $G_{d_{12}} \equiv G_{d_{12}}(g)$ and $G_{d_{12}, d_{13}, d_{23}} \equiv G_{d_{12}, d_{13}, d_{23}}(g)$ (resp. by $\tilde{G}_{d_{12}}$ and $\tilde{G}_{d_{12}, d_{13}, d_{23}}$ ) the two- and three-point functions of general (resp. bipartite) planar maps.

3.1. Two-point function of general maps. The two- and three-point functions may be expressed in terms of a number of generating functions for suitably defined well-labelled objects which we will introduce along this paper as we need them. A first basic ingredient is the generating function $T_{s} \equiv T_{s}(g)(s>0)$ of $(s)^{+}$-well-labelled maps, enumerated with a weight $g$ per edge, with a marked corner at a vertex labelled 0 . Here, by $(s)^{+}$-well-labelled map, we mean a well-labelled map with a single face $f$ (i.e., a tree) such that $\min (f) \geq-s+1$.

Introducing the notation

$$
[s]_{x} \equiv 1-x^{s},
$$

the following expression for $T_{s}$ has been known for quite a while, obtained by various techniques [5, 10]

$$
\begin{aligned}
& T_{s}=T \frac{[s]_{x}[s+3]_{x}}{[s+1]_{x}[s+2]_{x}} \\
& \text { where } T=\frac{1+4 x+x^{2}}{1+x+x^{2}} \text { and } g=x \frac{1+x+x^{2}}{\left(1+4 x+x^{2}\right)^{2}} .
\end{aligned}
$$

In the above parametrization of $g$ (which is symmetric under $x \rightarrow 1 / x$ ) and in similar expressions below, we always pick for $x$ the solution having modulus less than 1 near $g=0$ (in particular $x=O(g)$ ). Note then that, for $s>0, T_{s}=1+O(g)$ with a first term 1 accounting for the "vertex-map" reduced to a single vertex with label 0 . Note also that the above expression for $T_{s}$ may formally be extended to the case $s=0$ as it yields $T_{0}=0$, which is the wanted result.

An explicit expression for the two-point function of general planar maps was already obtained in [2, 7]. It follows from a bijective coding of maps with two marked vertices at distance $d_{12}$ by $(s)$-well-labelled maps (well-labelled maps with a single face $f$ satisfying $\min (f)=-s+1)$ with $s=d_{12}$, with a marked vertex labelled 0 which is not a local max. It was found that, for $d_{12} \geq 1$

$$
G_{d_{12}}=\log \left(\frac{1+g T_{s} T_{s+1}}{1+g T_{s-1} T_{s}}\right)=\log \left(\frac{\left([s+1]_{x}\right)^{3}[s+3]_{x}}{[s]_{x}\left([s+2]_{x}\right)^{3}}\right) \quad \text { with } s=d_{12} .
$$




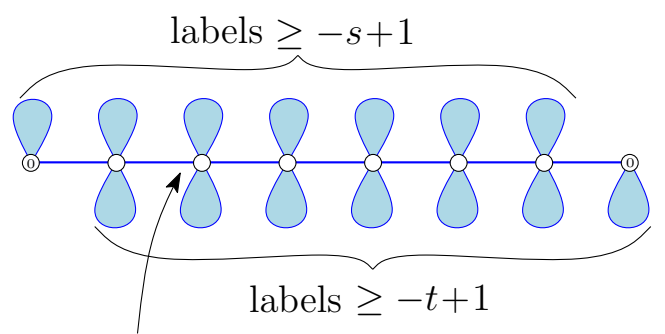

spine labels $\geq 0$

type A: no (1)

type B: at least one (1)—

FiguRE 6. Schematic picture of an $(s, t)^{+}$-well-labelled chain. The blobs represent attached well-labelled subtrees. The labels of the spine-vertices are 0 at the extremities of the spine and non-negative in-between. The chain is of type $\mathrm{A}$ if it has no spine-edge with labels $0-0$, and of type $\mathrm{B}$ otherwise.

Our new bijections (A) and (B) of Theorem 2 provide two alternative routes to recover this two-point function. This new approach is instructive as it will lead us to introduce and evaluate new generating functions which will turn out to be useful to later compute the three-point function.

The first generating function of interest is that, $N_{s, t} \equiv N_{s, t}(g)$, of $(s, t)^{+}$well-labelled chains of type A, enumerated with a weight $g$ per edge, defined as follows: by well-labelled chain, we mean a well-labelled tree made of a distinguished linear (oriented) spine of some arbitrary length, with additional subtrees attached to the spine-vertices (see Figure 6 for an illustration). We have an $(s, t)^{+}$-well-labelled chain if (i) the labels of the spine-vertices are 0 at the extremities of the spine and non-negative in-between and (ii) the vertices of the subtrees attached to the first extremity of the spine and to the left of all inner spine-vertices have a label $\geq-s+1$ and the vertices of the subtrees attached to the last extremity of the spine and to the right of all inner spine-vertices have a label $\geq-t+1$. Finally an $(s, t)^{+}$-well-labelled chain is said to be of type $A$ if there is no spine-edge with labels $0-0$. If the spine has length 0 , i.e., reduces to a single vertex, we decide by convention not to attach any subtree at all to this vertex: this configuration receives a weight 1 accordingly. The above definition of $N_{s, t}$ holds for $s, t>0$ and we extend it for convenience by setting $N_{s, 0}=N_{0, t}=N_{0,0}=1$.

Similarly, we may introduce the generating function $O_{s, t} \equiv O_{s, t}(g)$ of $(s, t)^{+}$-well-labelled chains of type $\mathrm{B}$ defined as $(s, t)^{+}$-well-labelled chains with at least one spine-edge with labels $0-0$ (again this definition holds for $s, t>0$ and we extend it by now setting $O_{s, 0}=$ $\left.O_{0, t}=O_{0,0}=0\right)$.

From the bijection (A) of Theorem 2 , when $d_{12} \geq 2, G_{d_{12}}$ is also the generating function of $(s, t)$-well-labelled maps of type $\mathrm{A}$, enumerated with a weight $g$ per edge, for any pair of positive integers $s$ and $t$ satisfying $s+t=d_{12}$. At this stage, it is useful to weaken the definition of $(s, t)$-well-labelled maps of type $\mathrm{A}$ and consider instead what we shall call $(s, t)^{+}$-well-labelled maps of type A, satisfying now the weaker conditions $\min \left(f_{1}\right) \geq-s+1$ and $\min \left(f_{2}\right) \geq-t+1$ for the labels incident to their faces $f_{1}$ and $f_{2}$. In other words, an $(s, t)^{+}$-well-labelled map is an $\left(s^{\prime}, t^{\prime}\right)$-well-labelled map with $0<s^{\prime} \leq s$ and $0<t^{\prime} \leq t$. Focusing on the cycle $\Gamma$ formed by their border-vertices and border-edges, we then remark that $(s, t)^{+}$-well-labelled maps of type A may be seen as cyclic sequences of elementary blocks (which consist of $(s, t)^{+}$-well-labelled chains of type A having no spine-label 0 between the extremities of its spine) while $(s, t)^{+}$-well-labelled chains of type A correspond to (linear) sequences of blocks of the same type. By a standard argument, we immediately deduce that 
the generating function for $(s, t)^{+}$-well-labelled maps of type $\mathrm{A}$ is given by $\log \left(N_{s, t}\right)$ and consequently, that that of $(s, t)$-well labelled maps of type $\mathrm{A}$ is given by $\Delta_{s} \Delta_{t} \log \left(N_{s, t}\right)$. Here $\Delta_{s}$ is the finite difference operator $\Delta_{s} f(s) \equiv f(s)-f(s-1)$. Applying $\Delta_{s}$ on the generating function $\log \left(N_{s, t}\right)$ indeed selects those configurations satisfying $-s+2>\min \left(f_{1}\right) \geq-s+1$, hence $\min \left(f_{1}\right)=-s+1$ and similarly, applying $\Delta_{t}$ imposes $\min \left(f_{2}\right)=-t+1$. The finite difference operators therefore ensure the passage from $(s, t)^{+}$-well-labelled objects to $(s, t)$ well-labelled ones. We shall use this standard trick in various occasions later in the paper. We deduce the relation, for $s, t>0$

$$
G_{d_{12}}=\Delta_{s} \Delta_{t} \log \left(N_{s, t}\right)=\log \left(\frac{N_{s, t} N_{s-1, t-1}}{N_{s-1, t} N_{s, t-1}}\right) \quad \text { with } s+t=d_{12}
$$

(note that this holds for $s=1$ and $t=1$ thanks to our convention $N_{s, 0}=N_{0, t}=N_{0,0}=1$ ).

To evaluate $N_{s, t}$, we may rely on a known formula for the generating function $X_{s, t}=$ $N_{s, t}+O_{s, t}$ of $(s, t)^{+}$-well-labelled chains of type A or B (i.e., with or without spine-edges of labels $0-0)$. This later generating function was indeed computed in [8, with the result

$$
X_{s, t}=\frac{[3]_{x}[s+1]_{x}[t+1]_{x}[s+t+3]_{x}}{[1]_{x}[s+3]_{x}[t+3]_{x}[s+t+1]_{x}}
$$

and satisfies the following recursion relation (obtained by decomposing the chain at its first return at label 0 along the spine)

$$
X_{s, t}=1+g T_{s} T_{t} X_{s, t}+g^{2} T_{s} T_{t} X_{s, t} T_{s+1} T_{t+1} X_{s+1, t+1} .
$$

Now, by a decomposition of the chain at the spine-edges with labels $0-0$, we may write the relation $X_{s, t}=N_{s, t} /\left(1-g T_{s} T_{t} N_{s, t}\right)$, or equivalently

$$
N_{s, t}=\frac{X_{s, t}}{1+g T_{s} T_{t} X_{s, t}} .
$$

Replacing $X_{s, t}$ by its value above, this leads to the particularly simple (and remarkably similar) expression

$$
N_{s, t}=\frac{[3]_{x}[s+2]_{x}[t+2]_{x}[s+t+3]_{x}}{[2]_{x}[s+3]_{x}[t+3]_{x}[s+t+2]_{x}} .
$$

Plugging this latter expression in (4), we get explicitely

$$
G_{d_{12}}=\log \left(\frac{\left([s+t+1]_{x}\right)^{3}[s+t+3]_{x}}{[s+t]_{x}\left([s+t+2]_{x}\right)^{3}}\right) \quad \text { with } s+t=d_{12},
$$

which reproduces precisely the previous formula (3), as wanted.

The knowledge of the generating function $N_{s, t}$ will be crucial in the derivation of the three-point function in the next section. It is interesting to note that its above expression (8) may be obtained in several alternative ways, without recourse to the known expression for $X_{s, t}$. These alternative approaches will prove useful when we shall discuss similar generating functions for which we cannot rely on known formulas.

First, we note that equating $a b$ initio (3) and (4) provides in return a constructive way of getting $N_{s, t}$. Indeed, it allows us to write

$$
\frac{N_{s, t} N_{s-1, t-1}}{N_{s-1, t} N_{s, t-1}}=\frac{R_{s+t}}{R_{s+t-1}}, \quad R_{u} \equiv 1+g T_{u} T_{u+1}=\frac{\left([2]_{x}\right)^{2}}{[1]_{x}[3]_{x}} \frac{[u+1]_{x}[u+3]_{x}}{\left([u+2]_{x}\right)^{2}},
$$

which is a double (in $s$ and $t$ ) recursion formula. Together with the conditions $N_{s, 0}=N_{0, t}=$ $N_{0,0}=1$, it leads to

$$
N_{s, t}=\prod_{u=1}^{s+t} R_{u} /\left(\prod_{u=1}^{s} R_{u} \prod_{u=1}^{t} R_{u}\right),
$$

which yields immediately (8) by replacing $R_{u}$ by its value. 


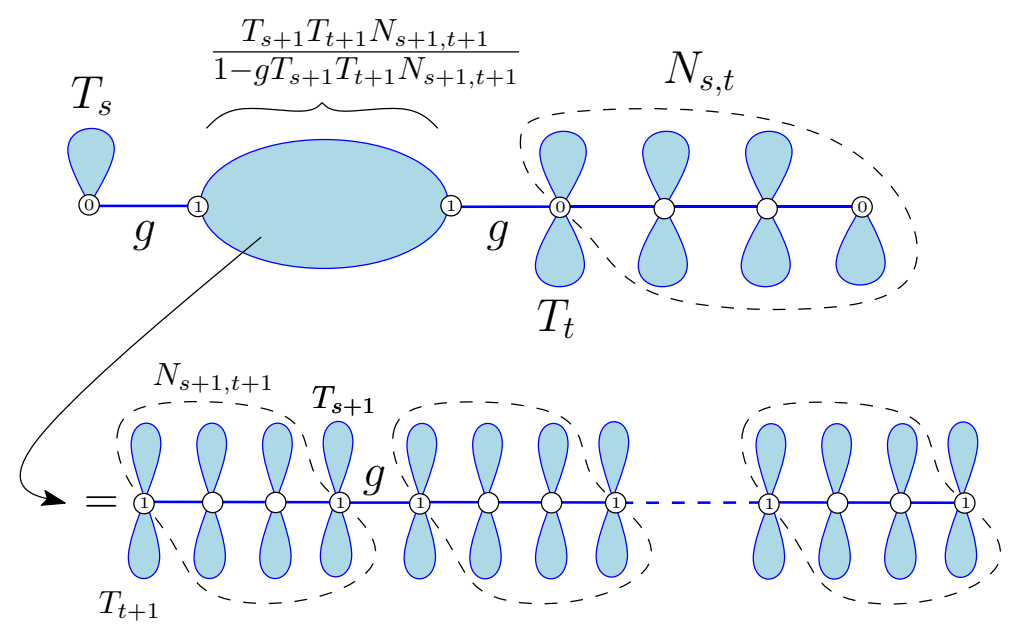

Figure 7. A schematic picture of the recursion relation for $N_{s, t}$, obtained by decomposing an $(s, t)^{+}$-well-labelled chain of type $\mathrm{A}$ at its first return at label 0 along the spine.

A second alternative, but non-constructive way of getting $N_{s, t}$ is by solving yet another recursion relation, which is the analog of (6), obtained by a simple decomposition of an $(s, t)^{+}$-well-labelled chain of type $\mathrm{A}$ at its first return at label 0 along the spine. This decomposition leads to (see Figure 7)

$$
N_{s, t}=1+g^{2} T_{s} T_{t} N_{s, t} \frac{T_{s+1} T_{t+1} N_{s+1, t+1}}{1-g T_{s+1} T_{t+1} N_{s+1, t+1}} .
$$

Indeed, when not reduced to a single vertex (weight 1 ), an $(s, t)^{+}$-well-labelled chain of type A has a spine made of a first $0-1$ edge (weight $g T_{s}$, including the attached subtree), a portion of spine with labels larger than or equal to 1 , then a first $1-0$ edge (weight $g T_{t}$ ) and a final portion which is itself an $(s, t)^{+}$-well-labelled chain of type A (weight $N_{s, t}$ ). The portion of spine with labels larger than or equal to 1 is, after a simple shift of labels by -1 , an $(s+1, t+1)^{+}$-well-labelled chain (of arbitrary type A or B) with two extra attached subtrees, as enumerated by $T_{s+1} T_{t+1} X_{s+1}=T_{s+1} T_{t+1} N_{s+1, t+1} /\left(1-g T_{s+1} T_{t+1} N_{s+1, t+1}\right)$. Note that eq. (12) may equivalently be obtained by simply plugging $X_{s, t}=N_{s, t} /\left(1-g T_{s} T_{t} N_{s, t}\right)$ into (6). Knowing the expression of $T_{s}$, this equation determines entirely $N_{s, t}$ as a power series in $g$. It is now a straightforward exercise to check that the above expression (8) does indeed solve this equation (and is such that $N_{s, t}=1+O(g)$ ) hence provides the correct expression for $N_{s, t}$. In the following, we shall recourse in several occasions to this method, i.e., write down a recursion relation and guess its (unique as a power series in $g$ ) solution.

We may now easily repeat the above arguments by using instead the bijection (B) of Theorem 2 For $d_{12} \geq 1, G_{d_{12}}$ is then identified with the generating function of $(s, t)$-welllabelled maps of type $\mathrm{B}$, enumerated with a weight $g$ per edge, for any pair of positive integers $s$ and $t$ such that $d_{12}=s+t-1$. Now, focusing on the cycle $\Gamma$ formed by their border-vertices and border-edges, $(s, t)^{+}$-well-labelled maps of type $\mathrm{B}$ are nothing but cyclic sequences where each elementary block is made of an edge with labels $0-0$ (weight $g$ ) followed by an $(s, t)^{+}$-well-labelled chain of type A with two extra attached subtrees (weight $\left.T_{s} T_{t} N_{s, t}\right)$. This allows us to write immediately

$$
\begin{aligned}
G_{d_{12}} & =\Delta_{s} \Delta_{t} \log \left(\frac{1}{1-g T_{s} T_{t} N_{s, t}}\right)=\Delta_{s} \Delta_{t} \log \left(\frac{X_{s, t}}{N_{s, t}}\right) \\
& =\log \left(\frac{X_{s, t} X_{s-1, t-1} N_{s-1, t} N_{s, t-1}}{X_{s-1, t} X_{s, t-1} N_{s, t} N_{s-1, t-1}}\right) .
\end{aligned}
$$


Replacing $X_{s, t}$ and $N_{s, t}$ by their expressions, we deduce

$$
G_{d_{12}}=\log \left(\frac{\left([s+t]_{x}\right)^{3}[s+t+2]_{x}}{[s+t-1]_{x}\left([s+t+1]_{x}\right)^{3}}\right) \quad \text { with } s+t-1=d_{12}
$$

in agreement with (3), as wanted.

To end this section, let us finally give an expression for the generating function $O_{s, t}$ of $(s, t)^{+}$-well-labelled chains of type B. It is obtained via $O_{s, t}=X_{s, t}-N_{s, t}$, from which we deduce

$$
O_{s, t}=x \frac{[3]_{x}[s]_{x}[t]_{x}\left([s+t+3]_{x}\right)^{2}}{[2]_{x}[s+3]_{x}[t+3]_{x}[s+t+1]_{x}[s+t+2]_{x}} .
$$

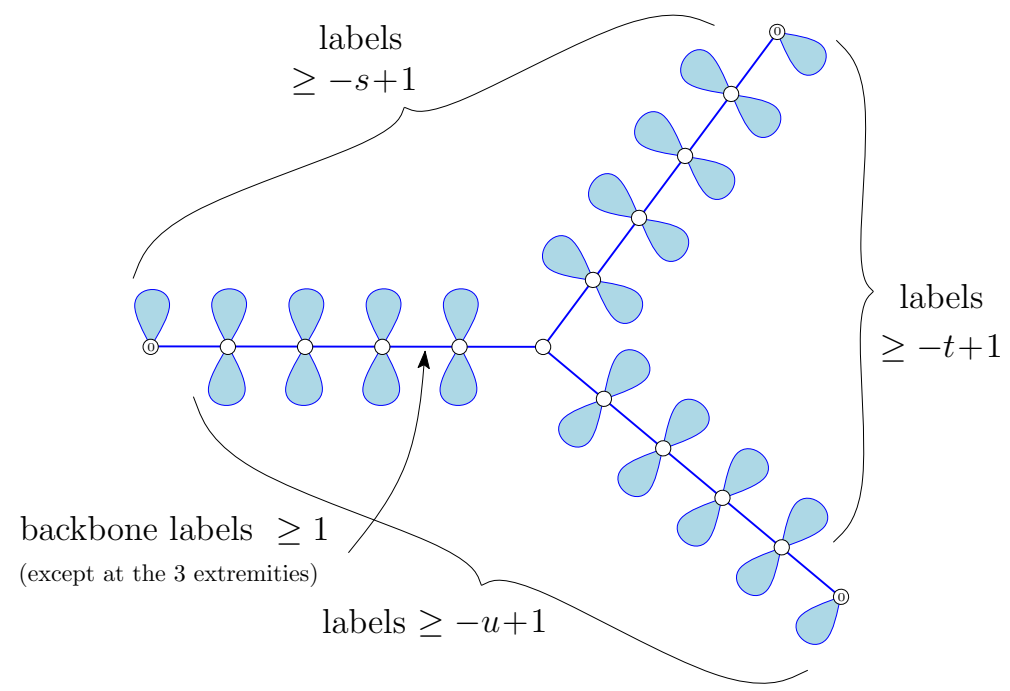

Figure 8. Schematic picture of an $(s, t, u)^{+}$-well-labelled Y-diagram. The blobs represent attached well-labelled subtrees. The label of the backbonevertices are 0 at the extremities of the three branches and positive inbetween.

3.2. Three-point function of general maps. We shall now use the bijections of Theorem 3 to derive an explicit formula for the three-point function of general planar maps. As a new tool, we shall need an expression for the generating function $Y_{s, t, u} \equiv Y_{s, t, u}(g)$ of $(s, t, u)^{+}$-well-labelled $Y$-diagrams defined as follows, for $s, t, u>0$ : by well-labelled Ydiagram, we mean a well-labelled tree with a distinguished backbone made of three branches (referred to as the first, second and third branch clockwise) of arbitrary lengths connected at a central vertex, with additional subtrees attached to the backbone-vertices (see Figure 8 for an illustration). The labels of the backbone vertices are required to be 0 at the extremities of the three branches and strictly positive in-between. We have an $(s, t, u)^{+}-$Y-diagram if, going clockwise around the backbone, the vertices of the subtrees attached to backbone-corners lying between the extremity of the first branch (extremity included) and that of the second branch (extremity excluded) have a label $\geq-s+1$, the vertices of the subtrees attached to backbone-corners lying between the extremity of the second branch (extremity included) and that of the third branch (extremity excluded) have a label $\geq-t+1$ and, finally, the vertices of the subtrees attached to backbone-corners lying between the extremity of the third branch (extremity included) and that of the first branch (extremity excluded) have a label $\geq-u+1$. Note that an $(s, t, u)^{+}$- well-labelled Y-diagram cannot contain edges with labels $0-0$ along its three branches since only the extremities of the branches carry a label 
0 . Note also that the lengths of the three branches may be zero simultaneously in with case the backbone of the Y-diagram reduces to a single vertex with label 0: as before, we then decide for convenience not to attach any subtree at all to this vertex and this configuration receives a weight 1 accordingly.

The generating function $Y_{s, t, u}$ was already introduced in 8 . There it was shown that it satisfies the following recursion relation, easily obtained by decomposing each branch of the Y-diagram at its first passage (starting from the central vertex) at label 1 along the branch

$$
Y_{s, t, u}=1+g^{3} T_{s} T_{t} T_{u} X_{s+1, t+1} X_{s+1, u+1} X_{t+1, u+1} T_{s+1} T_{t+1} T_{u+1} Y_{s+1, t+1, u+1} .
$$

Knowing $T_{s}$ and $X_{s, t}$, this equation determines $Y_{s, t, u}$ entirely as a power series in $g$. The following explicit solution was then found in 8

$$
Y_{s, t, u}=\frac{[s+3]_{x}[t+3]_{x}[u+3]_{x}[s+t+u+3]_{x}}{[3]_{x}[s+t+3]_{x}[t+u+3]_{x}[u+s+3]_{x}} .
$$

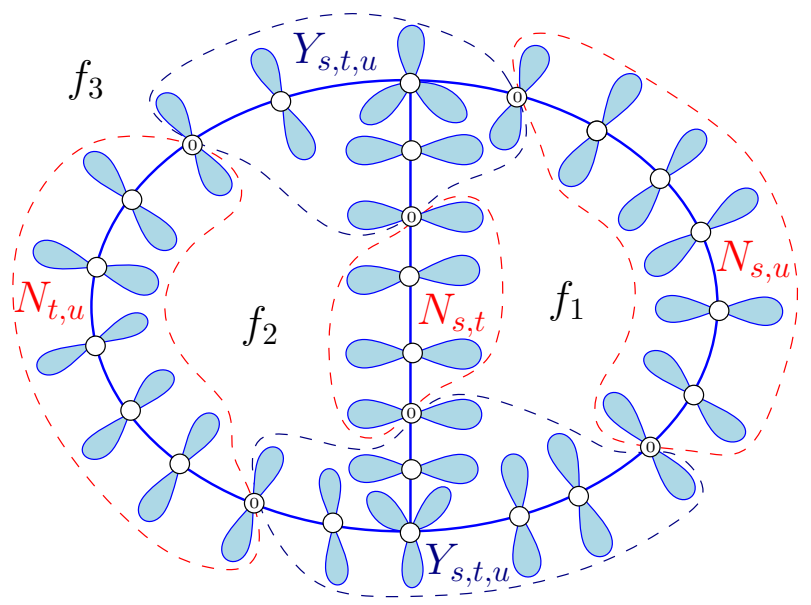

FigurE 9. Schematic picture of an $(s, t, u)^{+}$-well-labelled map. The blobs represent attached well-labelled subtrees. The map is decomposed into five pieces, two of them enumerated by $Y_{s, t, u}$ and the last three by $N_{s, t}, N_{s, u}$ and $N_{t, u}$ respectively (see text).

We may now evaluate the three-point function $G_{d_{12}, d_{13}, d_{23}}$ of general maps. Let us start with the case where $d_{12}+d_{13}+d_{23}$ is even and $d_{12}, d_{13}$ and $d_{23}$ satisfy strict triangular inequalities (we will return later to the case where triangular inequalities are not strict). As in the statement of Theorem 3 (A), we then set

$$
d_{12}=s+t, \quad d_{13}=s+u, \quad d_{23}=t+u,
$$

where $s, t$ and $u$ are three positive integers. From the bijection (A) of Theorem $3, G_{d_{12}, d_{13}, d_{23}}$ is identified with the generating function of $(s, t, u)$-well-labelled maps of type $\mathrm{A}$, which allows us to write

$$
G_{d_{12}, d_{13}, d_{23}}=\Delta_{s} \Delta_{t} \Delta_{u} N_{s, t} N_{s, u} N_{t, u} Y_{s, t, u}^{2} .
$$

Indeed, introducing $(s, t, u)^{+}$-well-labelled maps of type $\mathrm{A}$ (defined as $(s, t, u)$-well-labelled maps of type $\mathrm{A}$ except for the weaker constraints $\min \left(f_{1}\right) \geq-s+1, \min \left(f_{2}\right) \geq-t+1$ and $\left.\min \left(f_{3}\right) \geq-u+1\right)$, these maps may be decomposed into five pieces by cutting their "backbone" at the first and last occurrence of a label 0 along each border between their three faces. More precisely, introducing as before the three subgraphs $\Gamma_{i j}$ of the map made of vertices and edges incident to the faces $f_{i}$ and $f_{j}(1 \leq i<j \leq 3), \Gamma_{12}, \Gamma_{13}$ and $\Gamma_{23}$ form 
a backbone made generically of three chains attached at their extremities to two "triplepoint" vertices (see Figure 9p. Since the map is of type A, each $\Gamma_{i j}$ carries at least a label 0 by definition. Cutting the chains at its first and last occurrence of such a label 0 (as encountered by going from one triple-point to the other) results in an $(s, t, u)^{+}$-well-labeled Y-diagrams and an $(s, u, t)^{+}$-well-labeled Y-diagrams (both enumerated by $Y_{s, t, u}$ ) and three chains: an $(s, t)^{+}$-well-labeled chain, an $(s, u)^{+}$-well-labelled chain and a $(t, u)^{+}$-well-labelled chain, all of type $A$, hence enumerated by $N_{s, t}, N_{s, u}$ and $N_{t, u}$ respectively. Finally, the passage from $(s, t, u)^{+}$-well-labelled maps to $(s, t, u)$-well-labelled maps is performed by the action of the finite difference operators $\Delta_{s}, \Delta_{t}$ and $\Delta_{u}$. As explained in [8] (in a similar calculation for quadrangulations), degenerate situations where the border between two of the three faces is reduced to a single vertex (so that the two triple-points coalesce) are properly enumerated by this formula. For instance, configurations whose $(1,2)$-border is a single vertex are enumerated by $\Delta_{s} \Delta_{t} \Delta_{u} N_{s, u} N_{t, u}$. This contribution to $G_{d_{12}, d_{13}, d_{23}}$ properly appears in 19 by picking the first term 1 in the expansion of both $N_{s, t}$ and $Y_{s, t, u}$.

Replacing $Y_{s, t, u}$ and $N_{s, t}$ by their explicit expressions, we obtain the following result:

Proposition 3 (Three-point function of general maps: even case). Given $d_{12}$, $d_{13}$ and $d_{23}$ three positive integers satisfying strict triangular inequalities, and such that $d_{12}+d_{13}+d_{23}$ is even, the three-point function $G_{d_{12}, d_{13}, d_{23}}$ is given by

$$
\begin{aligned}
& G_{d_{12}, d_{13}, d_{23}}=\Delta_{s} \Delta_{t} \Delta_{u} F_{s, t, u}^{e v e n} \\
& F_{s, t, u}^{e v e n}=\frac{[3]_{x}\left([s+2]_{x}[t+2]_{x}[u+2]_{x}[s+t+u+3]_{x}\right)^{2}}{\left([2]_{x}\right)^{3}[s+t+2]_{x}[t+u+2]_{x}[u+s+2]_{x}[s+t+3]_{x}[t+u+3]_{x}[u+s+3]_{x}} \\
& \text { with } s, t, u \text { as in } \text { (18). }
\end{aligned}
$$

Let us now discuss the case where triangular inequalities are not strict by setting for instance $u=0$ in (18), in which case $d_{13}+d_{23}=d_{12}$ and $v_{3}$ lies on a geodesic path between $v_{1}$ and $v_{2}$. As explained in Remark 3, such tri-pointed maps are in bijection with $(s, t)$-welllabelled maps of type A with a marked border-vertex with label 0 . This marking transforms de facto (by a simple cut) the $(s, t)$-well-labelled map of type $\mathrm{A}$ into an $(s, t)$-well-labelled chain of type $\mathrm{A}$, as enumerated by $\Delta_{s} \Delta_{t} N_{s, t}$. We deduce

$$
G_{d_{13}+d_{23}, d_{13}, d_{23}}=\Delta_{s} \Delta_{t} N_{s, t} \quad \text { with } s=d_{13}, t=d_{23} .
$$

Note that this latter expression is precisely that given by 20 if we formally set $F_{s, t,-1}^{\text {even }}=0$ so that $\left.\Delta_{u} F_{s, t, u}^{\text {even }}\right|_{u=0}=F_{s, t, 0}^{\text {even }}=N_{s, t}$.

Let us now come to the case where $d_{12}+d_{13}+d_{23}$ is odd and, as in the statement of Theorem 3 (B), set

$$
d_{12}=s+t-1, \quad d_{13}=s+u-1, \quad d_{23}=t+u-1
$$

where $s, t$ and $u$ are positive integers. From the bijection (B) of Theorem $3, G_{d_{12}, d_{13}, d_{23}}$ is now identified with the generating function of $(s, t, u)$-well-labelled maps of type $\mathrm{B}$, which allows us to write

$$
G_{d_{12}, d_{13}, d_{23}}=\Delta_{s} \Delta_{t} \Delta_{u} O_{s, t} O_{s, u} O_{t, u} Y_{s, t, u}^{2}
$$

Replacing $Y_{s, t, u}$ and $O_{s, t}$ by their explicit expressions, we deduce

Proposition 4 (Three-point function of general maps: odd case). Given $d_{12}, d_{13}$ and $d_{23}$ three positive integers satisfying triangular inequalities, and such that $d_{12}+d_{13}+d_{23}$ is odd, 
the three-point function $G_{d_{12}, d_{13}, d_{23}}$ is given by

$$
\begin{aligned}
& G_{d_{12}, d_{13}, d_{23}}=\Delta_{s} \Delta_{t} \Delta_{u} F_{s, t, u}^{o d d} \\
& F_{s, t, u}^{o d d}=x^{3} \frac{[3]_{x}\left([s]_{x}[t]_{x}[u]_{x}[s+t+u+3]_{x}\right)^{2}}{\left([2]_{x}\right)^{3}[s+t+1]_{x}[t+u+1]_{x}[u+s+1]_{x}[s+t+2]_{x}[t+u+2]_{x}[u+s+2]_{x}} \\
& \text { with } s, t, u \text { as in } 22 .
\end{aligned}
$$

As a simple application of our formulas, let us compute for instance the first terms in the small $g$ expansion of $G_{2,2,2}$ (three vertices at pairwise distances $d_{12}=d_{13}=d_{23}=2$ ) and $G_{1,1,1}$ (three vertices at pairwise distances $d_{12}=d_{13}=d_{23}=1$ ). Both are obtained by setting $s=t=u=1$, respectively in (20) and (24). From the relation between $g$ and $x$ in (2), we deduce the expansion

$$
x=g+7 g^{2}+59 g^{3}+544 g^{4}+5289 g^{5}+53256 g^{6}+549771 g^{7}+5782105 g^{8}+\ldots
$$

Plugging this expansion in 20 and 24 yields

$$
\begin{aligned}
& G_{2,2,2}=2 g^{3}+39 g^{4}+558 g^{5}+7123 g^{6}+86139 g^{7}+1011954 g^{8}+\ldots \\
& G_{1,1,1}=g^{3}+15 g^{4}+174 g^{5}+1867 g^{6}+19482 g^{7}+201450 g^{8}+\ldots
\end{aligned}
$$

whose first terms (of order $g^{3}$ and $g^{4}$ ) are easily recovered by a simple inspection.

3.3. Two- and three-point functions of bipartite maps. We may easily repeat the arguments of Sects. 3.1 and 3.2 to obtain the two- and three-point functions of bipartite planar maps. In practice, we simply have to consider the same generating functions as above restricted to the subclass of very-well-labelled objects. Our first basic ingredient is therefore the generating function $\tilde{T}_{s} \equiv \tilde{T}_{s}(g)(s>0)$ of $(s)^{+}$-very-well-labelled maps, enumerated with a weight $g$ per edge, with a marked corner at a vertex labelled 0 . The following expression for $\tilde{T}_{s}$ was derived in 5

$$
\begin{aligned}
& \tilde{T}_{s}=\tilde{T} \frac{[s]_{x}[s+4]_{x}}{[s+1]_{x}[s+3]_{x}} \\
& \text { where } \tilde{T}=\frac{(1+x)^{2}}{1+x^{2}} \text { and } g=x \frac{1+x^{2}}{(1+x)^{4}} .
\end{aligned}
$$

As explained in [7, we may deduce from $\tilde{T}_{s}$ an explicit formula for the two-point function of bipartite planar maps

$$
\tilde{G}_{d_{12}}=\log \left(\frac{1+g \tilde{T}_{s} \tilde{T}_{s+1}}{1+g \tilde{T}_{s-1} \tilde{T}_{s}}\right)=\log \left(\frac{\left([s+1]_{x}\right)^{2}[s+4]_{x}}{[s]_{x}\left([s+3]_{x}\right)^{2}}\right) \quad \text { with } s=d_{12} .
$$

Let us now see how to recover this formula in the framework of bi-pointed maps. From Theorem 2. $\tilde{G}_{d_{12}}$ with $d_{12}=s+t$ is identified with the generating function of $(s, t)$-verywell-labelled maps of type A, enumerated with a weight $g$ per edge. Note that being of type A simply amounts here to demanding that the minimum label over all border vertices in the map is 0 , and that there are no $(s, t)$-very-well-labelled maps of type B. As for $(s, t)^{+}$-verywell-labelled chains, they are automatically of type A: if we insist in defining the very-welllabelled analogs $\tilde{N}_{s, t}, \tilde{O}_{s, t}$ and $\tilde{X}_{s, t}$ of $N_{s, t}, O_{s, t}$ and $N_{s, t}$, we must then set

$$
\tilde{N}_{s, t}=\tilde{X}_{s, t} \quad \text { and } \quad \tilde{O}_{s, t}=0
$$

so that, in practice, we only have to deal with a single generating function $\tilde{X}_{s, t}$, that of $(s, t)^{+}$-very-well labelled chains. By the same argument as in Sect. 3.1, we may now write 
the two-point function as

$$
\tilde{G}_{d_{12}}=\Delta_{s} \Delta_{t} \log \left(\tilde{X}_{s, t}\right)=\log \left(\frac{\tilde{X}_{s, t} \tilde{X}_{s-1, t-1}}{\tilde{X}_{s-1, t} \tilde{X}_{s, t-1}}\right) \quad \text { with } s+t=d_{12} .
$$

Comparing with 28 , this leads to the identification

$$
\tilde{X}_{s, t}=\prod_{u=1}^{s+t} \tilde{R}_{u} /\left(\prod_{u=1}^{s} \tilde{R}_{u} \prod_{u=1}^{t} \tilde{R}_{u}\right), \quad \tilde{R}_{u} \equiv 1+g \tilde{T}_{u} \tilde{T}_{u+1}=\frac{[2]_{x}[3]_{x}}{[1]_{x}[4]_{x}} \frac{[u+1]_{x}[u+4]_{x}}{[u+2]_{x}[u+3]_{x}}
$$

hence to the explicit expression

$$
\tilde{X}_{s, t}=\frac{[4]_{x}[s+2]_{x}[t+2]_{x}[s+t+4]_{x}}{[2]_{x}[s+4]_{x}[t+4]_{x}[s+t+2]_{x}} .
$$

As a check of consistency, we now argue that $\tilde{X}_{s, t}$ is, alternatively, entirely determined as a power series in $g$ by the recursion

$$
\tilde{X}_{s, t}=1+g^{2} \tilde{T}_{s} \tilde{T}_{t} \tilde{X}_{s, t} \tilde{T}_{t+1} \tilde{T}_{s+1} \tilde{X}_{s_{1}, t+1},
$$

obtained by decomposing the chain at its first return to 0 along the spine. It is a simple exercise to check that 32 actually solves this equation, as wanted.

Coming now to the three-point function $\tilde{G}_{d_{12}, d_{13}, d_{23}}$ of bipartite planar maps, we set again (recall that the sum $d_{12}+d_{13}+d_{23}$ is necessarily even in a bipartite map)

$$
d_{12}=s+t, \quad d_{13}=s+u, \quad d_{23}=t+u
$$

with $s, t$ and $u$ positive integers (we assume here strict triangular inequalities). To get $\tilde{G}_{d_{12}, d_{13}, d_{23}}$, we now need an expression for the generating function $\tilde{Y}_{s, t, u} \equiv \tilde{Y}_{s, t, u}(g)$ of $(s, t, u)^{+}$-very-well-labelled Y-diagrams (which form the very-well labelled subclass of $(s, t, u)^{+}$well-labelled Y-diagrams). Since no expression was known so far for $\tilde{Y}_{s, t, u}$, we had to recourse to the same guessing approach as in [8]. It is easy to write down a recursion relation for $\tilde{Y}_{s, t, u}$ of the same type as $(16)$, obtained again by decomposing each branch of an $(s, t, u)^{+}$ very-well-labelled Y-diagram at its first passage at label 1. It is in practice the same as (16) with $T_{s}$ and $X_{s, t}$ replaced by their tilde counterparts, i.e.,

$$
\tilde{Y}_{s, t, u}=1+g^{3} \tilde{T}_{s} \tilde{T}_{t} \tilde{T}_{u} \tilde{X}_{s+1, t+1} \tilde{X}_{s+1, u+1} \tilde{X}_{t+1, u+1} \tilde{T}_{s+1} \tilde{T}_{t+1} \tilde{T}_{u+1} \tilde{Y}_{s+1, t+1, u+1}
$$

and determines $\tilde{Y}_{s, t, u}$ entirely as a power series in $g$. We have been able to guess the solution of this equation, which has the slightly more involved expression (now a sum of two terms)

$$
\begin{aligned}
& \tilde{Y}_{s, t, u}=\frac{[s+4]_{x}[t+4]_{x}[u+4]_{x}}{[3]_{x}[4]_{x}[s+2]_{x}[t+2]_{x}[u+2]_{x}[s+t+4]_{x}[t+u+4]_{x}[u+s+4]_{x}} \times \\
& \quad \times\left(x[3]_{x}[s+1]_{x}[t+1]_{x}[u+1]_{x}[s+t+u+5]_{x}+[1]_{x}[s+3]_{x}[t+3]_{x}[u+3]_{x}[s+t+u+3]_{x}\right) .
\end{aligned}
$$

By the same argument as in Sect. 3.2, we obtain directly:

Proposition 5 (Three-point function of bipartite maps). Given $d_{12}, d_{13}$ and $d_{23}$ three positive integers satisfying strict triangular inequalities, and such that $d_{12}+d_{13}+d_{23}$ is even, the three-point function $\tilde{G}_{d_{12}, d_{13}, d_{23}}$ is given by

$$
\begin{aligned}
& \tilde{G}_{d_{12}, d_{13}, d_{23}}=\Delta_{s} \Delta_{t} \Delta_{u} \tilde{F}_{s, t, u} \\
& \tilde{F}_{s, t, u}=\tilde{X}_{s, t} \tilde{X}_{s, u} \tilde{X}_{t, u} \tilde{Y}_{s, t, u}^{2} \\
(37)= & \frac{[4]_{x}\left(x[3]_{x}[s+1]_{x}[t+1]_{x}[u+1]_{x}[s+t+u+5]_{x}+[1]_{x}[s+3]_{x}[t+3]_{x}[u+3]_{x}[s+t+u+3]_{x}\right)^{2}}{\left([2]_{x}\right)^{3}\left([3]_{x}\right)^{2}[s+t+2]_{x}[t+u+2]_{x}[u+s+2]_{x}[s+t+4]_{x}[t+u+4]_{x}[u+s+4]_{x}}
\end{aligned}
$$

with $s, t, u$ as in 34 . 
As before, the case where triangular inequalities are not strict requires a special attention: setting again $u=0$ for instance, we now arrive at

$$
\tilde{G}_{d_{13}+d_{23}, d_{13}, d_{23}}=\Delta_{s} \Delta_{t} \tilde{X}_{s, t} \quad \text { with } s=d_{13}, t=d_{23} .
$$

Again this latter expression coincides with that given by 37 if we formally set $\tilde{F}_{s, t,-1}=0$ so that $\left.\Delta_{u} \tilde{F}_{s, t, u}\right|_{u=0}=\tilde{F}_{s, t, 0}=\tilde{X}_{s, t}$.

A simple application of (37) is the small $g$ expansion of $\tilde{G}_{2,2,2}$ (three vertices at pairwise distances $d_{12}=d_{13}=d_{23}=2$ ) obtained by setting $s=t=u=1$ in (37). From the relation between $g$ and $x$ in 27 , we deduce the expansion

$$
x=g+4 g^{2}+21 g^{3}+124 g^{4}+782 g^{5}+5144 g^{6}+34845 g^{7}+241196 g^{8}+\ldots
$$

Plugging this expansion in (37) yields

$$
\tilde{G}_{2,2,2}=2 g^{3}+21 g^{4}+174 g^{5}+1336 g^{6}+9942 g^{7}+72966 g^{8} \ldots
$$

whose first terms (of order $g^{3}$ and $g^{4}$ ) are easily recovered by inspection.

\section{Bivariate Two- And three-Point FunCtions}

We can refine our analysis of the two- and three-point functions by keeping track of both the numbers of edges and faces of the maps. More precisely, we may compute the bivariate two- and three-point functions $G_{d_{12}}(g, z)$ and $G_{d_{12}, d_{13}, d_{23}}(g, z)$ for general planar maps (and their tilde analogs for bipartite maps) enumerated with both a weight $g$ per edge and a weight $z$ per face (and with a factor $1 / k$ in case of $k$-fold symmetry). As we did before in the univariate case, we shall omit in the following the arguments $g$ and $z$ in all the encountered generating functions, and write for instance $G_{d_{12}}$ and $G_{d_{12}, d_{13}, d_{23}}$ for short. All the generating functions discussed in this section are implicitly understood as bivariate generating functions, depending on both $g$ and $z$.

4.1. General maps. In the various bijections of Sect. 2, the faces of the bi- or tri-pointed maps at hand are in one-to-one correspondence with local max of the associated well-labelled maps. Recall that a local max is a vertex whose label is not smaller than that of any of its neighbours. Such a local max will now be assigned an additional weight $z$. Our first input is thus, for $s>0$, the generating function $T_{s} \equiv T_{s}(g, z)$ of $(s)^{+}$-well-labelled maps, enumerated with a weight $g$ per edge and a weight $z$ per local max, and with a marked corner at a vertex (the root vertex) labelled 0. As was done in 2, 7, it is useful to also introduce the generating function $U_{s} \equiv U_{s}(g, z)$ of the same objects but where the root vertex is weighted by 1 irrespectively of whether or not this vertex is a local max. Introducing the notation

$$
[s]_{x, \alpha} \equiv 1-\alpha x^{s},
$$

the following expressions for $T_{s}$ and $U_{s}$ were obtained in [2, 7 ]

$$
\begin{aligned}
& T_{s}=T \frac{[s]_{x, 1}[s+3]_{x, \alpha^{2}}}{[s+1]_{x, \alpha}[s+2]_{x, \alpha}}, \quad U_{s}=U \frac{[s]_{x, 1}[s+3]_{x, \alpha}}{[s+1]_{x, 1}[s+2]_{x, \alpha}}, \\
& \text { where } T=\frac{\alpha(1-x)^{2}\left(1+x+\alpha x-6 \alpha x^{2}+\alpha x^{3}+\alpha^{2} x^{3}+\alpha^{2} x^{4}\right)}{(1-\alpha x)^{3}\left(1-\alpha x^{3}\right)}, \\
& U=\frac{1+x+\alpha x-6 \alpha x^{2}+\alpha x^{3}+\alpha^{2} x^{3}+\alpha^{2} x^{4}}{(1-\alpha x)\left(1-\alpha x^{3}\right)}, \\
& g=\frac{x(1-\alpha x)^{3}\left(1-\alpha x^{3}\right)}{\left(1+x+\alpha x-6 \alpha x^{2}+\alpha x^{3}+\alpha^{2} x^{3}+\alpha^{2} x^{4}\right)^{2}} \\
& z=\frac{\alpha(1-x)^{3}\left(1-\alpha^{2} x^{3}\right)}{(1-\alpha x)^{3}\left(1-\alpha x^{3}\right)} .
\end{aligned}
$$


The bivariate two-point function was then computed, with result

$$
G_{d_{12}}=\log \left(\frac{1+g U_{s} T_{s+1}}{1+g U_{s-1} T_{s}}\right)=\log \left(\frac{\left([s+1]_{x, \alpha}\right)^{3}[s+3]_{x, \alpha}}{[s]_{x, \alpha}\left([s+2]_{x, \alpha}\right)^{3}}\right) \quad \text { with } s=d_{12} .
$$

Alternatively, the bijection (A) of Theorem 2 allows us to identify $G_{d_{12}}$ with $d_{12}=s+t$ with the generating function of $(s, t)$-well-labelled maps of type $\mathrm{A}$, enumerated with a weight $g$ per edge and $z$ per local max. Introducing the bivariate generating function $N_{s, t} \equiv N_{s, t}(g, z)$ of $(s, t)^{+}$-well-labelled chains of type $\mathrm{A}$ (with the convention that the configuration reduced to a single spine vertex receives the weight 1 ), we note that, as before, $(s, t)^{+}$-well-labelled maps of type $\mathrm{A}$ are simply enumerated by $\log \left(N_{s, t}\right)$. Indeed, $(s, t)^{+}$-well-labelled chains of type $\mathrm{A}$ and $(s, t)^{+}$-well-labelled maps of type $\mathrm{A}$ correspond again to sequences and cyclic sequences of the same elementary blocks, corresponding to $(s, t)^{+}$-well-labelled chains of type A having no spine label 0 between the extremities of their spine, now enumerated with the additional weight $z$ per local max. Here it is crucial to realize that, upon gluing (linearly or cyclically) the blocks, the (local max or not) nature of the gluing vertices is not affected since these vertices, with label 0 , are never local max within objects of type A. This allows us to write as before

$$
G_{d_{12}}=\Delta_{s} \Delta_{t} \log \left(N_{s, t}\right)=\log \left(\frac{N_{s, t} N_{s-1, t-1}}{N_{s-1, t} N_{s, t-1}}\right) \quad \text { with } s+t=d_{12},
$$

(with the convention $N_{s, 0}=N_{0, t}=N_{0,0}=1$ ) and, by comparing with the expression (43), to obtain the formula

$$
N_{s, t}=\frac{[3]_{x, \alpha}[s+2]_{x, \alpha}[t+2]_{x, \alpha}[s+t+3]_{x, \alpha}}{[2]_{x, \alpha}[s+3]_{x, \alpha}[t+3]_{x, \alpha}[s+t+2]_{x, \alpha}} .
$$
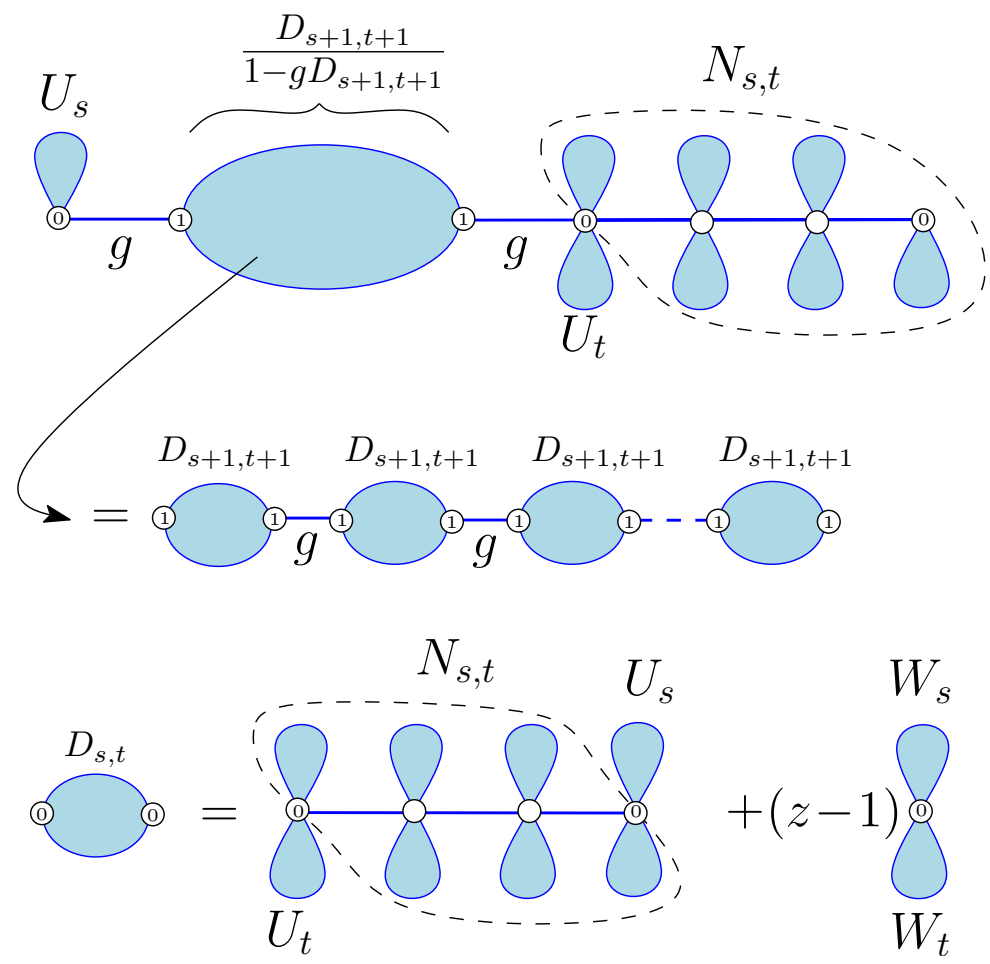

FiguRE 10. Recursion relation for $N_{s, t}$ obtained by decomposing an $(s, t)^{+}$well-labelled chain of type $\mathrm{A}$ at its first return at label 0 along the spine (see text). 
As a test of consistency, we may write down a recursion relation for $N_{s, t}$, the bivariate analog of (12), illustrated in Figure 10. It now reads

$$
N_{s, t}=1+g^{2} U_{s} U_{t} N_{s, t} \frac{D_{s+1, t+1}}{1-g D_{s+1, t+1}}
$$

where

$$
D_{s, t}=U_{s} U_{t} N_{s, t}+(z-1) W_{s} W_{t}, \quad W_{s}=\frac{U_{s}}{1+g U_{s} T_{s+1}} .
$$

Indeed, when not reduced to a single spine vertex (weight 1 ), an $(s, t)^{+}$-well-labelled chain of type $\mathrm{A}$ has a spine made of a first $0-1$ edge (weight $g U_{s}$ with the attached subtree since the vertex labelled 0 on the spine is not a local max), a portion of spine with labels larger than or equal to 1 , then a first $1-0$ edge (weight $g U_{t}$ since the vertex labelled 0 is not a local max) and a final portion which is itself an $(s, t)^{+}$-well-labelled chain of type A (weight $\left.N_{s, t}\right)$. The portion of spine with labels larger than or equal to 1 is, after a simple shift of labels by -1 , an $(s+1, t+1)^{+}$-well-labelled chain of type A or B with two extra attached subtrees. By a simple decomposition of this chain at each edge with labels $1-1$, it is now enumerated by $D_{s+1, t+1} /\left(1-g D_{s+1, t+1}\right)$ where $D_{s, t}$ enumerates $(s, t)^{+}$-well-labelled chains of type A with two extra attached trees, with the slight modification that, when the chain is reduced to a single spine vertex, and when the two extra attached trees are such that this spine vertex is a local max, then the spine vertex should receive a weight $z$ instead of 1 . This leads to the above expression (47) for $D_{s, t}$ with a first term $U_{s} U_{t} N_{s, t}$ giving a weight 1 to the chain reduced to a single spine vertex, then corrected by a term $(z-1) W_{s} W_{t}$ accounting for the case where the single spine vertex in a local max. Here $W_{s}$ enumerates $(s)^{+}$-welllabelled maps with a marked corner at a vertex (the root vertex) having label 0 and being a local max. By a simple canonical decomposition of an arbitrary $(s)^{+}$-well-labelled map with a marked corner at a vertex labelled 0 (this map is a planted tree enumerated by $U_{s}$ ) by marking each of its descending subtrees with root label 1 (each such descending subtree is enumerated by $g T_{s+1}$ and the part between two such subtrees is enumerated by $W_{s}$ ), we have $U_{s}=W_{s} /\left(1-g T_{s+1} W_{s}\right)$, hence the expression (47) for $W_{s}$.

Using the explicit forms 42 , we arrive at

$$
g D_{s, t}=\frac{\alpha x[1]_{x, 1}[s]_{x, 1}[t]_{x, 1}[s+t+3]_{x, \alpha^{2}}}{[2]_{x, \alpha}[s+1]_{x, \alpha}[t+1]_{x, \alpha}[s+t+2]_{x, \alpha}}
$$

and it is a straightforward exercise to check that (45) actually solves 46 .

We may alternatively evaluate the two-point function by using instead the bijection (B) of Theorem 2, writing now $d_{12}=s+t-1$ and identifying $G_{d_{12}}$ with the bivariate generating function of $(s, t)$-well-labelled maps of type B enumerated with a weight $g$ per edge and $z$ per local max. As before, $(s, t)^{+}$-well-labelled maps of type B are cyclic sequences where each elementary block consists of an edge with labels $0-0$ (weight $g$ ) followed by an $(s, t)^{+}$-welllabelled chain of type A with two extra attached subtrees, as enumerated by $D_{s, t}$ (again if the chain is reduced to a single vertex and the two extra attached trees are such that this vertex is a local max, then it should receive the weight $z$ ). This leads immediately to

$$
\begin{aligned}
G_{d_{12}} & =\Delta_{s} \Delta_{t} \log \left(\frac{1}{1-g D_{s, t}}\right) \\
& =\Delta_{s} \Delta_{t} \log \left(\frac{[2]_{x, \alpha}[s+1]_{x, \alpha}[t+1]_{x, \alpha}[s+t+2]_{x, \alpha}}{[1]_{x, \alpha}[s+2]_{x, \alpha}[t+2]_{x, \alpha}[s+t+1]_{x, \alpha}}\right) \\
& =\log \left(\frac{\left([s+t]_{x, \alpha}\right)^{3}[s+t+2]_{x, \alpha}}{[s+t-1]_{x, \alpha}\left([s+t+1]_{x, \alpha}\right)^{3}}\right) \quad \text { with } s+t-1=d_{12} .
\end{aligned}
$$

in agreement with 43 , as wanted.

Let us now compute the bivariate three-point function $G_{d_{12}, d_{13}, d_{23}}=G_{d_{12}, d_{13}, d_{23}}(g, z)$, starting with the case where $d_{12}+d_{13}+d_{23}$ is even (and $d_{12}, d_{13}$ and $d_{23}$ satisfy strict 
triangular inequalities), using the parametrization (18). In this case, we have as before

$$
G_{d_{12}, d_{13}, d_{23}}=\Delta_{s} \Delta_{t} \Delta_{u} N_{s, t} N_{s, u} N_{t, u} Y_{s, t, u}^{2},
$$

with $N_{s, t}$ as in (45) and where $Y_{s, t, u}$ is now the bivariate generating function for $(s, t, u)^{+}$ well-labelled Y-diagrams (here again we use the convention that the Y-diagram reduced to a single backbone vertex receives the weight 1 ). Once more, it is crucial to realize that, upon gluing the different pieces to get an $(s, t, u)^{+}$-well-labelled map of type A, the (local max or not) nature of the gluing vertices is not affected since these vertices, with label 0 , are never local max. To evaluate $Y_{s, t, u}$, we can write a recursion relation analog to (16), obtained by decomposing each branch of the Y-diagram at its first passage at label 1 (from the central vertex) along the branch. It reads

$$
\begin{aligned}
& Y_{s, t, u}= 1+g^{3} U_{s} U_{t} U_{u} \frac{1}{1-g D_{s+1, t+1}} \frac{1}{1-g D_{s+1, u+1}} \frac{1}{1-g D_{t+1, u+1}} \times \\
& \times\left(N_{s+1, t+1} N_{s+1, u+1} N_{t+1, u+1} U_{s+1} U_{t+1} U_{u+1} Y_{s+1, t+1, u+1}\right. \\
&\left.+(z-1) W_{s+1} W_{t+1} W_{u+1}\right),
\end{aligned}
$$

with the following interpretation: going towards the central vertex, each branch is formed of a first $0-1$ edge (weight $g U_{s}, g U_{t}$ and $g U_{u}$ respectively for the three branches, the three vertices with label 0 being not local max) followed by a portion of backbone until we reach the last $1-1$ edge on the branch (if there is no $1-1$ edge on the branch, this portion is empty). This portion is enumerated by $1 /\left(1-g D_{s+1, t+1}\right), 1 /\left(1-g D_{s+1, u+1}\right)$ and $1 /\left(1-g D_{t+1, u+1}\right)$ respectively on the three branches. After this last $1-1$ edge (or after the first $0-1$ edge if the portion is empty), the branch continues with a portion without $1-1$ edges, as enumerated by $U_{t+1} N_{s+1, t+1}, U_{u+1} N_{t+1, u+1}$ and $U_{s+1} N_{s+1, u+1}$ respectively until the vertex with label 1 closest to the central vertex is reached (note the presence of the terms $U_{t+1}, U_{u+1}$ or $U_{s+1}$ accounting for the tree attached to the right of the first vertex in this portion). The remaining part is (by shifting the labels by -1$)$ an $(s+1, t+1, u+1)^{+}$ well-labelled Y-diagram, enumerated by $Y_{s+1, t+1, u+1}$, hence the first term in the parentheses in (51). In the above enumeration, we have assumed that the extremities of the last portion without $1-1$ edge were not local max. This is true except when the central vertex itself has label 1, when the last portion without $1-1$ edge is of length 0 for each branch, and when the trees attached to the central vertex are such that this vertex has no neighbours with larger labels. This explains the correction $(z-1) W_{s+1} W_{t+1} W_{u+1}$ in the parentheses.

Although equation (51) may appear slightly involved, its solution is, remarkably, the simplest possible generalization of (17) that we may think of, namely

$$
Y_{s, t, u}=\frac{[s+3]_{x, \alpha}[t+3]_{x, \alpha}[u+3]_{x, \alpha}[s+t+u+3]_{x, \alpha}}{[3]_{x, \alpha}[s+t+3]_{x, \alpha}[t+u+3]_{x, \alpha}[u+s+3]_{x, \alpha}} .
$$

Plugging this expression in (50), we obtain:

Proposition 6 (Bivariate three-point function of general maps: even case). Given $d_{12}$, $d_{13}$ and $d_{23}$ three positive integers satisfying strict triangular inequalities, and such that $d_{12}+d_{13}+d_{23}$ is even, the bivariate three-point function $G_{d_{12}, d_{13}, d_{23}}$ is given by

$$
\begin{aligned}
& G_{d_{12}, d_{13}, d_{23}}=\Delta_{s} \Delta_{t} \Delta_{u} F_{s, t, u}^{e v e n} \\
& F_{s, t, u}^{e v e n}=\frac{[3]_{x, \alpha}\left([s+2]_{x, \alpha}[t+2]_{x, \alpha}[u+2]_{x, \alpha}[s+t+u+3]_{x, \alpha}\right)^{2}}{\left([2]_{x, \alpha}\right)^{3}[s+t+2]_{x, \alpha}[t+u+2]_{x, \alpha}[u+s+2]_{x, \alpha}[s+t+3]_{x, \alpha}[t+u+3]_{x, \alpha}[u+s+3]_{x, \alpha}}
\end{aligned}
$$


This formula deals with situations where the $d_{12}, d_{23}$ and $d_{31}$ satisfy strict triangular inequalities. If not, say $d_{13}+d_{23}=d_{12}$, one may easily verify that the relation $G_{d_{13}+d_{23}, d_{13}, d_{23}}=\Delta_{s} \Delta_{t} N_{s, t}$ (with $d_{13}=s$ and $d_{23}=t$ ) remains valid in the bivariate case, now with $N_{s, t}$ given by 45 .

The case where $d_{12}+d_{13}+d_{23}$ is odd requires the bivariate generating function of $(s, t, u)$ well-labelled maps of type B, with $s, t$ and $u$ as in $(22)$. The generating function of $(s, t, u)^{+}-$ well-labelled maps of type B is easily obtained by decomposing these maps into five pieces upon cutting their backbone at the first and last occurrence of an edge with labels $0-0$ along each border between their three faces. This yields

(54) $F_{s, t, u}^{\mathrm{odd}}=g^{3} \frac{1}{1-g D_{s, t}} \frac{1}{1-g D_{s, u}} \frac{1}{1-g D_{t, u}}\left(N_{s, t} N_{s, u} N_{t, u} U_{s} U_{t} U_{u} Y_{s, t, u}+(z-1) W_{s} W_{t} W_{u}\right)^{2}$.

We leave the proof of this formula to the reader, who will recognize the same basic building blocks as in the derivation of (51). This leads to:

Proposition 7 (Bivariate three-point function of general maps: odd case). Given $d_{12}, d_{13}$ and $d_{23}$ three positive integers satisfying triangular inequalities, and such that $d_{12}+d_{13}+d_{23}$ is odd, the bivariate three-point function $G_{d_{12}, d_{13}, d_{23}}$ is given by

$$
\begin{gathered}
G_{d_{12}, d_{13}, d_{23}}=\Delta_{s} \Delta_{t} \Delta_{u} F_{s, t, u}^{o d d} \\
F_{s, t, u}^{o d d}=x^{3} \frac{[3]_{x, \alpha}\left(\alpha[s]_{x, 1}[t]_{x, 1}[u]_{x, 1}[s+t+u+3]_{x, \alpha^{2}}\right)^{2}}{\left([2]_{x, \alpha}\right)^{3}[s+t+1]_{x, \alpha}[t+u+1]_{x, \alpha}[u+s+1]_{x, \alpha}[s+t+2]_{x, \alpha}[t+u+2]_{x, \alpha}[u+s+2]_{x, \alpha}} \\
\text { with } s, t, u \text { as in } 22 .
\end{gathered}
$$

Again, as a simple application of our formulas, let us revisit the small $g$ expansion of $G_{2,2,2}$ and $G_{1,1,1}$. From the relation between $g, z, x$ and $\alpha$ in (42), we deduce the expansions

$$
\begin{aligned}
x=g+ & (2+5 z) g^{2}+\left(5+31 z+23 z^{2}\right) g^{3}+\left(14+153 z+275 z^{2}+102 z^{3}\right) g^{4}+ \\
& +\left(42+696 z+2170 z^{2}+1938 z^{3}+443 z^{4}\right) g^{5} \\
& +\left(132+3042 z+14212 z^{2}+21937 z^{3}+12035 z^{4}+1898 z^{5}\right) g^{6}+\ldots \\
\alpha=z+ & 3 z(1-z) g+3 z(1-z)(4+z) g^{2}+z(1-z)\left(49+51 z+4 z^{4}\right) g^{3} \\
& +3 z(1-z)\left(67+150 z+62 z^{2}+2 z^{3}\right) g^{4} \\
& +3 z(1-z)\left(275+1038 z+955 z^{2}+219 z^{3}+3 z^{4}\right) g^{5} \\
& +z(1-z)\left(3384+18965 z+29747 z^{2}+15651 z^{3}+2310 z^{4}+11 z^{5}\right) g^{6}+\ldots
\end{aligned}
$$

and consequently

$$
G_{2,2,2}=2 z g^{3}+3 z(4+9 z) g^{4}+18 z\left(3+15 z+13 z^{2}\right) g^{5}+z\left(220+1795 z+3453 z^{2}+1655 z^{3}\right) g^{6}+\ldots
$$

$$
G_{1,1,1}=z^{2} g^{3}+3 z^{2}(2+3 z) g^{4}+3 z^{2}\left(9+30 z+19 z^{2}\right) g^{5}+z^{2}\left(110+600 z+845 z^{2}+312 z^{3}\right) g^{6}+\ldots
$$

whose first terms (of order $g^{3}$ and $g^{4}$ ) may be checked by a simple inspection.

It is interesting to look at the $z \rightarrow 0$ limit of our three-point function. From 42 , this limit is reached by letting $\alpha \rightarrow 0$, in which case

$$
g=\frac{x}{(1+x)^{2}}+O(\alpha), \quad z=(1-x)^{3} \alpha+O\left(\alpha^{2}\right) .
$$

Expanding $F_{s, t, u}^{\text {even }}$ at first order in $\alpha$, we find

$$
F_{s, t, u}^{\text {even }}=\frac{x^{2}\left(3-x-2 x^{s}-2 x^{t}-2 x^{u}+(1+x)\left(x^{s+t}+x^{t+u}+x^{u+s}\right)-2 x^{s+t+u+1}\right)}{(1-x)^{3}} z+O\left(z^{2}\right)
$$




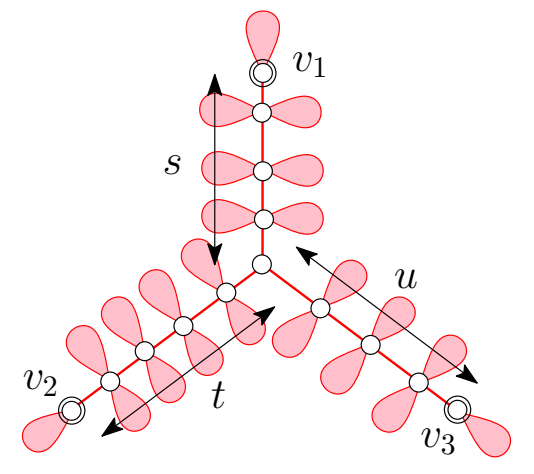

FIGURE 11. Schematic picture of a tri-pointed tree with pairwise distances $d_{12}=s+t, d_{13}=s+u$ and $d_{23}=t+u$. The blobs represent attached subtrees.

so that, from (53), in the case where $d_{12}+d_{13}+d_{23}$ is even

$$
\begin{aligned}
& G_{d_{12}, d_{13}, d_{23}}=2 x^{s+t+u} z+O\left(z^{2}\right)=2 x^{\frac{d_{12}+d_{13}+d_{23}}{2}} z+O\left(z^{2}\right) \\
& \text { with } x=g\left(\frac{1-\sqrt{1-4 g}}{2 g}\right)^{2} .
\end{aligned}
$$

This corresponds, as it should, to the three-point function of planar trees enumerated with a weight $g$ per edge. It enumerates tri-pointed trees of the form displayed in Figure 11. with a backbone made of three branches of respective lengths $s, t$ and $u$ as in (18). A weight $g$ is attached to each backbone edge and a weight Cat $(g)=(1-\sqrt{1-4 g}) /(2 g)$ (the Catalan function) to each backbone corner, to account for the possible subtrees attached to the backbone. This leads to the above formula since the backbone has $s+t+u$ edges and $2(s+t+u)$ corners. The prefactor 2 in 60$)$ comes from the two possible (clockwise or counterclockwise) cyclic orders in which the three distinguished vertices $v_{1}, v_{2}$ and $v_{3}$ may appear in the plane.

If $d_{12}+d_{13}+d_{23}$ is odd, we obtain from

$$
F_{s, t, u}^{\text {odd }}=\frac{x^{3}\left(\left(1-x^{s}\right)\left(1-x^{t}\right)\left(1-x^{u}\right)\right)^{2}}{(1-x)^{6}} z^{2}+O\left(z^{3}\right)
$$

the leading order in $z$

$$
G_{d_{12}, d_{13}, d_{23}}=x^{s+t+u} \frac{\left(2-x^{s}-x^{s-1}\right)\left(2-x^{t}-x^{t-1}\right)\left(2-x^{u}-x^{u-1}\right)}{(1-x)^{3}} z^{2}+O\left(z^{3}\right)
$$

$$
\text { with } x=g\left(\frac{1-\sqrt{1-4 g}}{2 g}\right)^{2} \text { and } s, t, u \text { as in } 22 \text {. }
$$

This now corresponds, as it should, to the enumeration of configurations of tri-pointed maps having two faces, which is the minimal number of faces in this case since the map cannot be bipartite. As displayed in Figure 12, such configurations are characterized by a backbone which forms a "geodesic triangle" between the three marked vertices and is made of an open triangular part with three attached "legs". The backbone is then completed by subtrees attached to all its corners and, as in the even case, the number of corners of the backbone is twice its number of edges so the correct enumeration is performed by simply assigning a weight $x=g \operatorname{Cat}^{2}(g)$ to each backbone edge. The lengths of the different portions of the backbone are constrained by the pairwise distances and we may distinguish three parts: a 


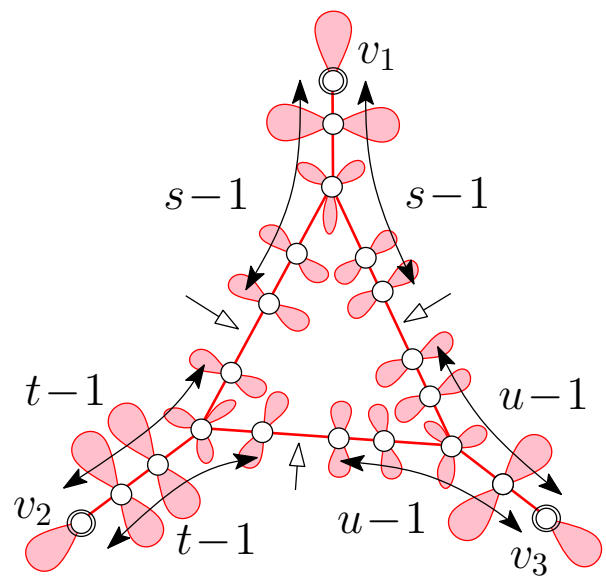

Figure 12. Schematic picture of a tri-pointed map with two faces and pairwise distances $d_{12}=s+t-1, d_{13}=s+u-1$ and $d_{23}=t+u-1$. The blobs represent attached subtrees. Suppressing the three edges marked by an open arrow cuts the map into three parts, corresponding to those backbone edges and vertices at distance $\leq s-1$ from vertex $v_{1}$ (resp. $\leq t-1$ from $v_{2}, \leq u-1$ from $\left.v_{3}\right)$. Each of these parts is partially unzipped, the zipped portion lying either inside or outside the open triangular part.

part made of those backbone-edges and vertices at distance less than or equal to $s-1$ from vertex $v_{1}$, a part made of those backbone-edges and vertices at distance less than or equal to $t-1$ from vertex $v_{2}$, a part made of those backbone-edges and vertices at distance less than or equal to $u-1$ from vertex $v_{3}$. The backbone is then the union of these three parts and of three remaining edges. Each part is partially unzipped, the zipped portion corresponding to one of the legs of the backbone. This allows us to write the generating function as $x^{3} Z_{s} Z_{t} Z_{u}$ where $Z_{s}$ properly enumerates the first part. If we call $i$ the length of its zipped portion, we find

$$
Z_{s}=\sum_{i=0}^{s-1}\left(2-\delta_{i, 0}\right) x^{i+2(s-1-i)}=x^{s-1} \frac{2-x^{s}-x^{s-1}}{1-x} .
$$

Note the factor of 2 whenever $i>0$. Indeed, having distinguished the three marked vertices, we may canonically differentiate between the two faces in the map and call one, say the interior and the other the exterior. Whenever the zipped portion has a non-zero length, we then must decide whether it lies in the interior or the exterior. There is no such choice for $i=0$. With this expression of $Z_{s}$, we recover the formula 62 .

4.2. Bipartite maps. Let us now come to the bivariate two- and three-point functions $\tilde{G}_{d_{12}}(g, z)$ and $\tilde{G}_{d_{12}, d_{13}, d_{23}}(g, z)$ of bipartite planar maps. As a first building block, we consider, for $s>0$, the bivariate generating function $\tilde{T}_{s} \equiv \tilde{T}_{s}(g, z)$ of $(s)^{+}$-very-well-labelled maps, enumerated with a weight $g$ per edge, $z$ per local max, and with a marked corner at a vertex labelled 0 . As before, we also introduce the generating function $\tilde{U}_{s}=\tilde{U}_{s}(g, z)$ of the same objects, but with a weight 1 for the root vertex even if it is a local max. Expressions 
for $\tilde{T}_{s}$ and $\tilde{U}_{s}$ were given in 7 .

$$
\begin{aligned}
& \tilde{T}_{s}=\tilde{T} \frac{[s]_{x, 1}[s+4]_{x, \alpha^{2}}}{[s+1]_{x, \alpha}[s+3]_{x, \alpha}}, \quad \tilde{U}_{s}=\tilde{U} \frac{[s]_{x, 1}[s+4]_{x, \alpha}}{[s+1]_{x, 1}[s+3]_{x, \alpha}}, \\
& \text { where } \tilde{T}=\frac{\alpha\left(1-x^{2}\right)^{2}\left(1-\alpha x^{2}\right)}{(1-\alpha x)^{2}\left(1-\alpha x^{4}\right)} \\
& \qquad \tilde{U}=\frac{(1+x)\left(1-\alpha x^{2}\right)^{2}}{(1-\alpha x)\left(1-\alpha x^{4}\right)} \\
& \qquad \begin{aligned}
g & =\frac{x(1-\alpha x)^{2}\left(1-\alpha x^{4}\right)}{(1+x)^{2}\left(1-\alpha x^{2}\right)^{3}} \\
z & =\frac{\alpha(1-x)^{2}\left(1-x^{2}\right)\left(1+\alpha x^{2}\right)}{(1-\alpha x)^{2}\left(1-\alpha x^{4}\right)}
\end{aligned}
\end{aligned}
$$

As explained in [7, the two-point function of bipartite planar maps is then

$$
\tilde{G}_{d_{12}}=\log \left(\frac{1+g \tilde{U}_{s} \tilde{T}_{s+1}}{1+g \tilde{U}_{s-1} \tilde{T}_{s}}\right)=\log \left(\frac{\left([s+1]_{x . \alpha}\right)^{2}[s+4]_{x, \alpha}}{[s]_{x, \alpha}\left([s+3]_{x, \alpha}\right)^{2}}\right) \quad \text { with } s=d_{12} .
$$

This formula may be recovered via Theorem 2 as $\tilde{G}_{d_{12}}$ is also, for any pair of positive $s$ and $t$ with $s+t=d_{12}$, the generating function of $(s, t)$-very-well-labelled maps of type A, enumerated with a weight $g$ per edge and $z$ per local max. Consider the bivariate generating function $\tilde{X}_{s, t} \equiv \tilde{X}_{s, t}(g, z)$ of $(s, t)^{+}$- very-well-labelled chains, we now have again the relation (30), which leads to the identification

$$
\tilde{X}_{s, t}=\frac{[4]_{x, \alpha}[s+2]_{x, \alpha}[t+2]_{x, \alpha}[s+t+4]_{x, \alpha}}{[2]_{x, \alpha}[s+4]_{x, \alpha}[t+4]_{x, \alpha}[s+t+2]_{x, \alpha}} .
$$

As easily checked, $\tilde{X}_{s, t}$ now solves the following recursion relation

$$
\tilde{X}_{s, t}=1+g^{2} \tilde{U}_{s} \tilde{U}_{t} \tilde{X}_{s, t}\left(\tilde{U}_{t+1} \tilde{U}_{s+1} \tilde{X}_{s_{1}, t+1}+(z-1) \tilde{W}_{s} \tilde{W}_{t}\right),
$$

with

(68) $\quad \tilde{W}_{s}=\frac{\tilde{U}_{s}}{1+g \tilde{U}_{s} \tilde{T}_{s+1}}$.

The bivariate generating function $\tilde{Y}_{s, t, u} \equiv \tilde{Y}_{s, t, u}(g, z)$ of very-well-labelled $(s, t, u)^{+}-\mathrm{Y}$ diagrams, with a weight $g$ per edge and $z$ per local max, may be obtained by solving the bivariate analog of (35). It now reads

$$
\begin{array}{r}
\tilde{Y}_{s, t, u}=1+g^{3} \tilde{U}_{s} \tilde{U}_{t} \tilde{U}_{u}\left(\tilde{X}_{s+1, t+1} \tilde{X}_{s+1, u+1} \tilde{X}_{t+1, u+1} \tilde{U}_{s+1} \tilde{U}_{t+1} \tilde{U}_{u+1} \tilde{Y}_{s+1, t+1, u+1}\right. \\
\left.+(z-1) \tilde{W}_{s+1} \tilde{W}_{t+1} \tilde{W}_{u+1}\right) .
\end{array}
$$

Again we were able to guess the (slightly involved) solution of this equation

$$
\begin{aligned}
& \tilde{Y}_{s, t, u}= \frac{[s+4]_{x, \alpha}[t+4]_{x, \alpha}[u+4]_{x, \alpha}}{[3]_{x, \alpha}[4]_{x, \alpha}[s+2]_{x, \alpha}[t+2]_{x, \alpha}[u+2]_{x, \alpha}[s+t+4]_{x, \alpha}[t+u+4]_{x, \alpha}[u+s+4]_{x, \alpha}} \times \\
& \times(70) \times\left(\alpha x[3]_{x, \alpha}[s+1]_{x, 1}[t+1]_{x, 1}[u+1]_{x, 1}[s+t+u+5]_{x, \alpha^{2}}\right. \\
&\left.+[1]_{x, \alpha}[s+3]_{x, \alpha}[t+3]_{x, \alpha}[u+3]_{x, \alpha}[s+t+u+3]_{x, \alpha}\right) .
\end{aligned}
$$

This yields 
Proposition 8 (Bivariate three-point function of bipartite maps). Given $d_{12}, d_{13}$ and $d_{23}$ three positive integers satisfying strict triangular inequalities, and such that $d_{12}+d_{13}+d_{23}$ is even, the three-point function $\tilde{G}_{d_{12}, d_{13}, d_{23}}$ is given by

$$
\begin{aligned}
& \tilde{G}_{d_{12}, d_{13}, d_{23}}=\Delta_{s} \Delta_{t} \Delta_{u} \tilde{F}_{s, t, u} \\
& \tilde{F}_{s, t, u}=\tilde{X}_{s, t} \tilde{X}_{s, u} \tilde{X}_{t, u} \tilde{Y}_{s, t, u}^{2} \\
& =\frac{[4]_{x, \alpha}}{\left([2]_{x, \alpha}\right)^{3}\left([3]_{x, \alpha}\right)^{2}[s+t+2]_{x, \alpha}[t+u+2]_{x, \alpha}[u+s+2]_{x, \alpha}[s+t+4]_{x, \alpha}[t+u+4]_{x, \alpha}[u+s+4]_{x, \alpha}} \\
& \begin{array}{r}
(71) \quad \\
\times\left(\alpha x[3]_{x, \alpha}[s+1]_{x, 1}[t+1]_{x, 1}[u+1]_{x, 1}[s+t+u+5]_{x, \alpha^{2}}\right. \\
\left.+[1]_{x, \alpha}[s+3]_{x, \alpha}[t+3]_{x, \alpha}[u+3]_{x, \alpha}[s+t+u+3]_{x, \alpha}\right)^{2} \\
\text { with } s, t, u \text { as in }(34) .
\end{array}
\end{aligned}
$$

In a situation where, say $u=0, \tilde{G}_{d_{12}, d_{13}, d_{23}}$ is still obtained via (38), now with the bivariate $\tilde{X}_{s, t}$.

From (71), we can get for instance the small $g$ expansion of $\tilde{G}_{2,2,2}$ (three vertices at pairwise distances $d_{12}=d_{13}=d_{23}=2$ ) by setting $s=t=u=1$ in 71 . From (64), we have the expansion

$$
\begin{aligned}
x= & g+2(1+z) g^{2}+\left(5+13 z+3 z^{2}\right) g^{3}+\left(14+66 z+40 z^{2}+4 z^{3}\right) g^{4}+ \\
& +\left(42+306 z+339 z^{2}+90 z^{3}+5 z^{4}\right) g^{5}+2\left(66+678 z+1168 z^{2}+572 z^{3}+85 z^{4}+3 z^{5}\right) g^{6}+\ldots \\
& \\
\alpha 2) & z+2 z(1-z) g+z(1-z)(8-z) g^{2}+32 z(1-z) g^{3}+3 z(1-z)(43+14 z) g^{4} \\
& +2 z(1-z)\left(261+214 z+26 z^{2}\right) g^{5}+z(1-z)\left(2116+3093 z+958 z^{2}+62 z^{3}\right) g^{6}+\ldots
\end{aligned}
$$

and consequently

$$
\tilde{G}_{2,2,2}=2 z g^{3}+3 z(4+3 z) g^{4}+6 z\left(9+16 z+4 z^{2}\right) g^{5}+z\left(220+667 z+399 z^{2}+50 z^{3}\right) g^{6}+\ldots
$$

whose first terms may be checked by a simple inspection.

As a final exercise, let us look at the $z \rightarrow 0$ limit of $\tilde{G}_{d_{12}, d_{13}, d_{23}}$. Letting $\alpha \rightarrow 0$ in 64 , we have

$$
g=\frac{x}{(1+x)^{2}}+O(\alpha), \quad z=(1-x)^{3}(1+x) \alpha+O\left(\alpha^{2}\right) .
$$

and we find for $\tilde{F}_{s, t, u}$ the same leading term (linear in $z$ ) as we found in the previous section for $F_{s, t, u}^{\text {even }}$, so that again

$$
\begin{aligned}
& \tilde{G}_{d_{12}, d_{13}, d_{23}}=2 x^{s+t+u} z+O\left(z^{2}\right)=2 x^{\frac{d_{12}+d_{13}+d_{23}}{2}} z+O\left(z^{2}\right) \\
& \text { with } x=g\left(\frac{1-\sqrt{1-4 g}}{2 g}\right)^{2} .
\end{aligned}
$$

We recover the three-point function of planar trees, which is of course not a surprise since trees are automatically bipartite.

\section{Applications}

5.1. Critical line. Throughout this section, we will enumerate maps with a fixed value of $z$, ranging from 0 to $\infty$. The limit of maps with a large number of edges may then be captured by looking at the singularities in the variable $g$ of the various generating functions at hand. More precisely, these generating functions become singular when $g$ reaches a critical value $g_{\text {crit }} \equiv g_{\text {crit }}(z)$ depending on $z$. The points $\left(z, g_{\text {crit }}(z)\right)$ define the so-called critical line in the 
$(z, g)$ plane. In the case of general planar maps, this critical line may be found by looking for instance at the singularities of the generating functions $T$ and $U$ introduced in 42 . These functions satisfy the following recursion relations (which follow directly from the definition of $T_{s}$ and $U_{s}$ as generating functions for $(s)^{+}$-well-labelled maps, which are particular instances of trees)

$$
E_{1} \equiv T-z-g\left(2 U T+T^{2}\right)=0, \quad E_{2} \equiv U-1-g\left(2 U T+U^{2}\right)=0 .
$$

The location of their singularities is obtained by writing

$$
0=\operatorname{det}\left(\begin{array}{cc}
\partial_{T} E_{1} & \partial_{T} E_{2} \\
\partial_{U} E_{1} & \partial_{U} E_{2}
\end{array}\right)=4 g^{2} U^{2}+4 g^{2} T^{2}+4 g^{2} U T-4 g U-4 g T+1,
$$

which upon setting $U=v / g$ and $T=\tau / g$ reads

$$
4 v^{2}+4 \tau^{2}+4 v \tau-4 v-4 \tau+1=0 .
$$

The values of $g_{\text {crit }}$ and $z$ are then recovered by writing $E_{1}=E_{2}=0$, namely

$$
g_{\mathrm{crit}}=v(1-v-2 \tau), \quad z=\frac{\tau(1-\tau-2 v)}{v(1-v-2 \tau)} .
$$

The ellipse (78) may be parametrized as

$$
v=\frac{2 r^{2}}{3\left(r^{2}+3\right)}, \quad \tau=\frac{(r-3)^{2}}{6\left(r^{2}+3\right)},
$$

which yields the following parametrization of the critical line

$$
z=\frac{(3-r)^{3}(r+1)}{16 r^{3}} \quad g_{\text {crit }}(z)=\frac{4 r^{3}}{3\left(r^{2}+3\right)^{2}},
$$

with $r$ varying from 3 down to 0 when $z$ varies from 0 to $\infty$ (and in particular $r=1$ for $z=1$ ). Note that going from $z$ to $1 / z$ corresponds in the parameter $r$ to performing the involution $r \rightarrow(3-r) /(1+r)$, and that $g_{\text {crit }}(1 / z)=z g_{\text {crit }}(z)$. This property is a direct consequence of the trivial bijection which associates to each map its dual map. For $z \rightarrow 0$, we find $g_{\text {crit }}(0)=1 / 4$, consistent with a number of trees with $n$ edges growing like $4^{n}$, while for $z=1$, we find $g_{\text {crit }}(1)=1 / 12$, consistent with a number of planar maps with $n$ edges growing like $12^{n}$. Finally, for $z \rightarrow \infty$, we find $g_{\text {crit }}(z) \sim 1 /(4 z)$ as expected by duality with the $z \rightarrow 0$ limit (the dominant configurations at large $z$ are dual to trees).

5.2. Scaling limit. All the generating functions for general maps introduced in this paper are singular, for a fixed $z$, when $g \rightarrow g_{\text {crit }}(z)$. The so-called scaling limit is then obtained by letting, for a fixed $z$, the weight $g$ tend to $g_{\text {crit }}(z)$ as

$$
g=g_{\text {crit }}(z)\left(1-\epsilon^{4}\right), \quad \epsilon \rightarrow 0
$$

and letting simultaneously all the distances between the marked vertices tend to $\infty$ as $1 / \epsilon$. In other word, it amounts to write, in addition to 82 ,

$$
d_{12}=\frac{\mathcal{D}_{12}}{\epsilon} \quad d_{13}=\frac{\mathcal{D}_{13}}{\epsilon} \quad d_{23}=\frac{\mathcal{D}_{23}}{\epsilon} \quad s=\frac{\mathcal{S}}{\epsilon} \quad t=\frac{\mathcal{T}}{\epsilon} \quad u=\frac{\mathcal{U}}{\epsilon}
$$

with $\mathcal{D}_{12}, \mathcal{D}_{13}, \mathcal{D}_{23}, \mathcal{S}, \mathcal{T}$ and $\mathcal{U}$ remaining finite when $\epsilon \rightarrow 0$. The leading order in $\epsilon$ of the various generating functions then defines what we shall call continuous scaling functions. The computation of these continuous scaling functions is in principle only a first step in getting the asymptotics of large maps. Some extra step is indeed required to extract from these functions properly normalized continuous canonical scaling functions corresponding now to genuine probability densities for renormalized distances $\mathcal{D}_{i j}=d_{i j} / n^{1 / 4}$ in an ensemble of maps with a fixed number $n$ of edges, in the limit $n \rightarrow \infty$. The reader is invited to consult [5] for instance for an explicit example of how to perform this second step. Still, as we shall now see, a number of large $n$ asymptotic results are directly readable from the continuous scaling functions themselves. 
From 42 , fixing simultaneously the parameters $z$ as in 81 and $g$ as in 82 is achieved by adjusting simultaneously $x$ and $\alpha$ as functions of $r$ and $\epsilon$. When $\epsilon \rightarrow 0$, both $x$ and $\alpha$ tend to 1 and we find in particular

$$
x=1-2 \gamma \epsilon+O\left(\epsilon^{2}\right), \quad \gamma=\sqrt{\frac{3(3-r) \sqrt{r^{2}+3}}{2 r(r+3)}} .
$$

The quantity $\gamma$ will be referred to as the scaling factor in the following, as it fixes the scale for distances in the various continuous generating functions that we shall encounter. Its value at $z=1(r=1)$ is $\sqrt{3 / 2}$. Letting $\epsilon \rightarrow 0$, we find the expansions (these expansions require in practice expanding both $x$ and $\alpha$ at a sufficiently large order in $\epsilon$, we skip the details here)

$$
\begin{aligned}
& N_{s, t}=\frac{6}{3+r}\left\{(1+\epsilon \chi(\mathcal{S}, \mathcal{T})\}+O\left(\epsilon^{2}\right), \frac{1}{1-g D_{s, t}}=\frac{3+r}{2 r}\{1+\epsilon \chi(\mathcal{S}, \mathcal{T})\}+O\left(\epsilon^{2}\right)\right. \\
& \text { where } \chi \text { satisfies } \partial_{\mathcal{S}} \partial_{\mathcal{T}} \chi(\mathcal{S}, \mathcal{T})=2 \gamma^{3} \frac{\cosh (\gamma(\mathcal{S}+\mathcal{T}))}{\sinh ^{3}(\gamma(\mathcal{S}+\mathcal{T}))} .
\end{aligned}
$$

Both expressions lead, via (44) and 49, to

$$
G_{d_{12}}=\epsilon^{3} \mathcal{G}\left(\mathcal{D}_{12}\right)+O\left(\epsilon^{4}\right), \quad \mathcal{G}\left(\mathcal{D}_{12}\right)=2 \gamma^{3} \frac{\cosh \left(\gamma \mathcal{D}_{12}\right)}{\sinh ^{3}\left(\gamma \mathcal{D}_{12}\right)}
$$

Here we recognize the continuous two-point function $\mathcal{G}\left(\mathcal{D}_{12}\right)$ found in [5] for quadrangulations, up to the $z$-dependent scaling factor $\gamma$, which appears both in the argument of the function, hence fixes the scale for $\mathcal{D}_{12}$, and in its normalization. Note that at $r=1$ $(\gamma=\sqrt{3 / 2})$, this normalization differs by a factor of 2 from that of quadrangulations. This is because there are asymptotically twice as many bi-pointed quadrangulations with $n$ faces as bi-pointed planar maps with $n$ edges. More generally, the normalization is simply inherited from the asymptotics of bi-pointed maps in the fixed $z$ ensemble. It is wiped out when extracting from $\mathcal{G}\left(\mathcal{D}_{12}\right)$ the continuous canonical two-point function (the probability density for $\mathcal{D}_{12}=d_{12} / n^{1 / 4}$ for maps with a fixed, large $n$ ), which thus differs from that of quadrangulations found in [5] only by the change of scale $\mathcal{D}_{12} \rightarrow \gamma \mathcal{D}_{12}$.

From 85, we also deduce

$$
\begin{gathered}
\Delta_{s} \Delta_{t} N_{s, t}=\epsilon^{3} \frac{6}{3+r} \mathcal{G}\left(\mathcal{D}_{12}\right)+O\left(\epsilon^{4}\right), \quad \Delta_{s} \Delta_{t} \frac{1}{1-g D_{s, t}}=\epsilon^{3} \frac{3+r}{2 r} \mathcal{G}\left(\mathcal{D}_{12}\right)+O\left(\epsilon^{4}\right), \\
\text { with } \mathcal{S}+\mathcal{T}=\mathcal{D}_{12} .
\end{gathered}
$$

Without any further calculation, we immediately deduce from these equations that $\Delta_{s} \Delta_{t} N_{s, t}$ and $\Delta_{s} \Delta_{t} 1 /\left(1-g D_{s, t}\right)$ have, up to an explicit normalization factor, the same large $n$ asymptotics as the two-point function $G_{d_{12}}$. From Remark 2, the reader will realize that, as bipointed map generating functions, $\Delta_{s} \Delta_{t} N_{s, t}$ with $s+t=d_{12}\left(\right.$ resp. $\Delta_{s} \Delta_{t} 1 /\left(1-g D_{s, t}\right)$ with $s+t-1=d_{12}$ ) differs from the two-point function $G_{d_{12}}$ only by the marking of an extra vertex (resp. an extra edge) belonging to a geodesic path between the two marked vertices, this extra vertex being at distance $s$ from the first vertex (resp. this extra edge being the $s$-th edge along the path). We may therefore interpret

$$
N_{\text {geod vertices }}=\frac{6}{3+r} \quad \text { and } \quad N_{\text {geod edges }}=\frac{3+r}{2 r}
$$

as the average numbers of geodesic vertices and geodesic edges between two far-away (in practice at a distance of order $n^{1 / 4}$ ) vertices, at a fixed distance (itself of order $n^{1 / 4}$ ) from the first vertex, in the ensemble of bi-pointed general planar maps with $n$ edges, in the limit $n \rightarrow \infty$. Note that these numbers do not depend on the position along the geodesic path in this limit. For $z=1(r=1)$, we find $N_{\text {geod vertices }}=3 / 2$ and $N_{\text {geod edges }}=2$. For $z \rightarrow 0$ $(r \rightarrow 3)$, we find $N_{\text {geod vertices }}=N_{\text {geod edges }}=1$, in agreement with the fact that the map then degenerates into a tree, with a single geodesic path between two given vertices. 
As for the three-point function, we find that both $F_{s, t, u}^{\mathrm{even}}$ and $F_{s, t, u}^{\mathrm{odd}}$ behave at leading order in $\epsilon$ as $\epsilon^{-2} \mathcal{F}(\mathcal{S}, \mathcal{T}, \mathcal{U})$ where

$$
\mathcal{F}(\mathcal{S}, \mathcal{T}, \mathcal{U})=\frac{3(3-r)^{2}}{2(3+r)^{3} \gamma^{2}}\left(\frac{2 \sinh (\gamma(\mathcal{S}+\mathcal{T}+\mathcal{U})) \sinh (\gamma \mathcal{S}) \sinh (\gamma \mathcal{T}) \sinh (\gamma \mathcal{U})}{\sinh (\gamma(\mathcal{S}+\mathcal{T})) \sinh (\gamma(\mathcal{T}+\mathcal{U})) \sinh (\gamma(\mathcal{U}+\mathcal{S}))}\right)^{2}
$$

so that

$$
G_{d_{12}, d_{13}, d_{23}}=\mathcal{G}\left(\mathcal{D}_{12}, \mathcal{D}_{13}, \mathcal{D}_{23}\right) \epsilon+O\left(\epsilon^{2}\right) \quad \text { with } \mathcal{G}=\partial_{\mathcal{S}} \partial_{\mathcal{T}} \partial_{\mathcal{U}} \mathcal{F}(\mathcal{S}, \mathcal{T}, \mathcal{U})
$$

and $\mathcal{D}_{12}=\mathcal{S}+\mathcal{T}, \mathcal{D}_{13}=\mathcal{S}+\mathcal{U}, \mathcal{D}_{23}=\mathcal{T}+\mathcal{U}$

Here again we recognize the continuous three-point function $\mathcal{G}\left(\mathcal{D}_{12}, \mathcal{D}_{13}, \mathcal{D}_{23}\right)$ found in 8 for quadrangulations, now however with a new scale for the distances $\mathcal{D}_{i j}$, fixed by the $z$ dependent scaling factor $\gamma$, and with a new normalization. This normalization is inherited from the asymptotics of tri-pointed maps and we may understand it as follows: when $s, t$ and $u$ tend to $\infty, F_{s, t, u}^{\text {even }}+F_{s, t, u}^{\text {odd }}$ tends to the generating function of tri-pointed planar maps, in the fixed $z$ ensemble, with no constraint on the pairwise distances between the three marked vertices. Letting accordingly $\mathcal{S}, \mathcal{T}$ and $\mathcal{U}$ tend to $\infty$ in $2 \mathcal{F}(\mathcal{S}, \mathcal{T}, \mathcal{U}$ ) (in which case the term within the big parentheses in 89 tends to 1 ), the normalization in front measures the $\epsilon^{-2}=1 / \sqrt{1-g / g_{\text {crit }}}$ singularity of the generating function of tri-pointed planar maps, hence leads to the following asymptotics, at large number $n$ of edges, of the "number" of tri-pointed maps (enumerated with a weight $z$ per face)

$$
\text { \#tri-pointed maps } \sim \frac{3(3-r)^{2}}{(3+r)^{3} \gamma^{2}} \frac{g_{\text {crit }}^{-n}}{\sqrt{\pi} n^{1 / 2}} .
$$

Understanding the normalization in 89 is therefore equivalent to understanding this asymptotics.

Now, from the expansions (see 7 for a precise meaning of these functions as map generating functions)

$$
\begin{aligned}
& \log (1+g U T)=\text { const. }-\frac{\gamma^{2}}{3} \epsilon^{2}+O\left(\epsilon^{3}\right), \\
& 1+2 g U T+g T^{2}=\text { const. }-\frac{(3+r)\left(3+r^{2}\right) \gamma^{2}}{12 r^{2}} \epsilon^{2}+O\left(\epsilon^{3}\right),
\end{aligned}
$$

we read the asymptotics of the numbers of bi-pointed and pointed-rooted (with a marked vertex and a marked oriented edge) planar maps at large number $n$ of edges

$$
\begin{aligned}
& \text { \#bi-pointed maps } \sim \frac{\gamma^{2}}{3} \frac{g_{\text {crit }}^{-n}}{2 \sqrt{\pi} n^{3 / 2}}, \\
& \text { \#pointed-rooted maps } \sim \frac{(3+r)\left(3+r^{2}\right) \gamma^{2}}{12 r^{2}} \frac{g_{\text {crit }}^{-n}}{2 \sqrt{\pi} n^{3 / 2}} .
\end{aligned}
$$

Both could have been obtained by starting from simply pointed maps and choosing an extra vertex in the first case or an extra oriented edge in the second case. Since there are $2 n$ choices of oriented edges, we deduce by comparison that, in an ensemble of planar maps with a fixed number of edges $n$, and with a fixed weight $z$ per face, the average number $n_{v} \equiv n_{v}(z)$ of vertices and that, $n_{f} \equiv n_{f}(v)=n+2-n_{v}$, of faces are, asymptotically at large $n$, given by

$$
n_{v} \sim \frac{8 r^{2}}{(3+r)\left(3+r^{2}\right)} n, \quad n_{f} \sim \frac{(1+r)(3-r)^{2}}{(3+r)\left(3+r^{2}\right)} n
$$

(these asymptotics should not depend on the precise ensemble, namely whether the maps are simply pointed, as in the above argument, multiply pointed, rooted or not). For $z=1,(r=$ 1), $n_{v} \sim n_{f} \sim n / 2$ as expected since by duality, there are on average as many faces as vertices. More generally, the expressions for $n_{v}$ and $n_{f}$ are exchanged under $r \rightarrow(3-r) /(1+r)$ (corresponding to $z \rightarrow 1 / z)$ as a consequence of duality. For $z \rightarrow 0(r \rightarrow 3), n_{v} \sim n$ 
as expected for trees (or more generally maps with a finite number of faces) which have asymptotically as many vertices as edges.

Returning to tri-pointed maps, they may be obtained similarly from bi-pointed maps by choosing an extra third vertex. This explains eventually their asymptotics via the identity

$$
\frac{8 r^{2}}{(3+r)\left(3+r^{2}\right)} n \times \frac{\gamma^{2}}{3} \frac{g_{\text {crit }}^{-n}}{2 \sqrt{\pi} n^{3 / 2}}=\frac{3(3-r)^{2}}{(3+r)^{3} \gamma^{2}} \frac{g_{\text {crit }}^{-n}}{\sqrt{\pi} n^{1 / 2}} .
$$

To conclude, if we now extract from 89 the continuous canonical three-point function (corresponding, in an ensemble of maps with a large fixed number $n$ of edges and with three marked vertices, to the probability density for prescribed pairwise renormalized distances $\mathcal{D}_{12}=d_{12} / n^{1 / 4}, \mathcal{D}_{13}=d_{13} / n^{1 / 4}$ and $\left.\mathcal{D}_{23}=d_{23} / n^{1 / 4}\right)$, all prefactors are wiped out by normalization so that this canonical continuous three-point function is exactly the same as that found in 8 for quadrangulations apart from a global change of scale of the renormalized distances $\mathcal{D} \rightarrow \gamma \mathcal{D}$. In particular, its $z$-dependence is entirely contained in the scaling factor $\gamma$ via (84).

5.3. Bipartite maps. Let us repeat our derivation of the critical line in the $(z, g)$ plane, now in the case of bipartite planar maps. To this end, we now look at the singularities of the generating functions $\tilde{T}$ and $\tilde{U}$ introduced in 64 . These functions satisfy the recursion relations

$$
\tilde{E}_{1} \equiv \tilde{T}-z-g 2 \tilde{U} \tilde{T}=0, \quad \tilde{E}_{2} \equiv \tilde{U}-1-g\left(\tilde{U} \tilde{T}+\tilde{U}^{2}\right)=0
$$

and the location of their singularities is obtained from

$$
0=\operatorname{det}\left(\begin{array}{cc}
\partial_{\tilde{T}} \tilde{E}_{1} & \partial_{\tilde{T}} \tilde{E}_{2} \\
\partial_{\tilde{U}} \tilde{E}_{1} & \partial_{\tilde{U}} \tilde{E}_{2}
\end{array}\right)=4 g^{2} U^{2}-4 g U-g T+1,
$$

which upon setting $\tilde{U}=\tilde{v} / g$ and $\tilde{T}=\tilde{\tau} / g$ reads

$$
4 \tilde{v}^{2}-4 \tilde{v}-\tilde{\tau}+1=0 .
$$

Writing $\tilde{E}_{1}=\tilde{E}_{2}=0$, the values of $g_{\text {crit }}$ and $z$ follow

$$
z=\frac{(1-2 \tilde{v})^{3}}{(3-4 \tilde{v}) \tilde{v}^{2}}, \quad g_{\text {crit }}=(3-4 \tilde{v}) \tilde{v}^{2},
$$

with $\tilde{v}$ varying from $1 / 2$ down to 0 when $z$ varies from 0 to $\infty$ (and in particular $\tilde{v}=1 / 4$ for $z=1)$. For $z \rightarrow 0$, we find again $g_{\text {crit }}(0)=1 / 4$, consistent with the fact that we enumerate trees, while for $z=1$, we find $g_{\text {crit }}(1)=1 / 8$, consistent with a number of planar bipartite maps with $n$ edges growing like $8^{n}$. Finally, for $z \rightarrow \infty$, we find $g_{\text {crit }}(z) \sim 1 / z$. Note that there is no duality symmetry for bipartite maps.

The scaling limit is again reached, for a fixed value of $z$, by letting $g$ tend to its critical value (99) exactly as in (82) and letting simultaneously all the distances between the marked vertices tend to $\infty$ as in (83). From (64), this amounts to again adjust $x$ and $\alpha$ as functions of $\tilde{v}$ and $\epsilon$ and, when $\epsilon \rightarrow 0$, we now find

$$
x=1-2 \gamma \epsilon+O\left(\epsilon^{2}\right), \quad \gamma=\sqrt{\frac{\sqrt{3}(1-2 \tilde{v})}{2 \sqrt{\tilde{v}(1-\tilde{v})}}} .
$$

The value of the scaling factor at $z=1(\tilde{v}=1 / 4)$ is now $\gamma=1$. Letting $\epsilon \rightarrow 0$, we find the expansion

$$
\begin{aligned}
& \tilde{X}_{s, t}=(3-4 \tilde{v})\left\{(1+\epsilon \tilde{\chi}(\mathcal{S}, \mathcal{T})\}+O\left(\epsilon^{2}\right)\right. \\
& \text { where } \tilde{\chi} \text { satisfies } \partial_{\mathcal{S}} \partial_{\mathcal{T}} \tilde{\chi}(\mathcal{S}, \mathcal{T})=4 \gamma^{3} \frac{\cosh (\gamma(\mathcal{S}+\mathcal{T}))}{\sinh ^{3}(\gamma(\mathcal{S}+\mathcal{T}))}
\end{aligned}
$$


and consequently

$$
\tilde{G}_{d_{12}}=\epsilon^{3} \tilde{\mathcal{G}}\left(\mathcal{D}_{12}\right)+O\left(\epsilon^{4}\right), \quad \tilde{\mathcal{G}}\left(\mathcal{D}_{12}\right)=4 \gamma^{3} \frac{\cosh \left(\gamma \mathcal{D}_{12}\right)}{\sinh ^{3}\left(\gamma \mathcal{D}_{12}\right)},
$$

where we recognize again the continuous two-point function of [5], up to the $z$-dependent scaling factor $\gamma$.

Looking at the prefactor in (101), we deduce also the average number of geodesic vertices between two far-away vertices, at some arbitrary but fixed distance from the first vertex

$$
N_{\text {geod vertices }}=3-4 \tilde{v} \text {. }
$$

The formula gives $N_{\text {geod vertices }}=2$ for $z=1(\tilde{v}=1 / 4)$ and $N_{\text {geod vertices }}=1$ for $z \rightarrow 0$ $(\tilde{v} \rightarrow 1 / 2)$, as expected for trees.

From the expansions (see [7] for the interpretation of these functions as bipartite map generating functions)

$$
\begin{aligned}
& \log (1+g \tilde{U} \tilde{T})=\text { const. }-\frac{2 \gamma^{2}}{3} \epsilon^{2}+O\left(\epsilon^{3}\right), \\
& 1+2 g \tilde{U} \tilde{T}=\text { const. }-\frac{4(1-\tilde{v}) \gamma^{2}}{3 \tilde{v}(3-4 \tilde{v})} \epsilon^{2}+O\left(\epsilon^{3}\right),
\end{aligned}
$$

we read the asymptotics of the numbers of bi-pointed and pointed-rooted bipartite planar maps at large number $n$ of edges

$$
\begin{aligned}
& \text { \#bi-pointed bipartite maps } \sim \frac{2 \gamma^{2}}{3} \frac{g_{\text {crit }}^{-n}}{2 \sqrt{\pi} n^{3 / 2}}, \\
& \text { \#pointed-rooted bipartite maps } \sim \frac{4(1-\tilde{v}) \gamma^{2}}{3 \tilde{v}(3-4 \tilde{v})} \frac{g_{\text {crit }}^{-n}}{2 \sqrt{\pi} n^{3 / 2}},
\end{aligned}
$$

with $g_{\text {crit }}$ as in $(99)$ and, by comparison, the asymptotics of the average numbers $n_{v}=n_{v}(z)$ of vertices and $n_{f}=n_{f}(z)$ of faces

$$
\text { (106) } \quad n_{v} \sim \tilde{v} \frac{3-4 \tilde{v}}{1-\tilde{v}} n, \quad n_{f} \sim \frac{(1-2 \tilde{v})^{2}}{1-\tilde{v}} n
$$

For $z=1(\tilde{v}=1 / 4), n_{v} \sim 2 n / 3$ and $n_{f} \sim n / 3$, as expected since, via a trivial bijection with Eulerian triangulations, bipartite maps have equally distributed numbers of faces, of vertices of even color and of vertices of odd color. For $z \rightarrow 0(\tilde{v} \rightarrow 1 / 2), n_{v} \sim n$, as expected.

Using

$$
\tilde{v} \frac{3-4 \tilde{v}}{1-\tilde{v}} n \times \frac{2 \gamma^{2}}{3} \frac{g_{\text {crit }}^{-n}}{2 \sqrt{\pi} n^{3 / 2}}=\frac{(1-2 \tilde{v})^{2}(3-4 \tilde{v})}{4(1-\tilde{v})^{2} \gamma^{2}} \frac{g_{\text {crit }}^{-n}}{\sqrt{\pi} n^{1 / 2}}
$$

for $\gamma$ as in 100 , the asymptotics of tri-pointed maps is then

(108) \#tri-pointed bipartite maps $\sim \frac{(1-2 \tilde{v})^{2}(3-4 \tilde{v})}{4(1-\tilde{v})^{2} \gamma^{2}} \frac{g_{\text {crit }}^{-n}}{\sqrt{\pi} n^{1 / 2}}$.

This is consistent with the expansion $\tilde{F}_{s, t, u} \sim \epsilon^{-2} \tilde{\mathcal{F}}(\mathcal{S}, \mathcal{T}, \mathcal{U})$ for which we find

(109) $\tilde{\mathcal{F}}(\mathcal{S}, \mathcal{T}, \mathcal{U})=\frac{(1-2 \tilde{v})^{2}(3-4 \tilde{v})}{4(1-\tilde{v})^{2} \gamma^{2}}\left(\frac{2 \sinh (\gamma(\mathcal{S}+\mathcal{T}+\mathcal{U})) \sinh (\gamma \mathcal{S}) \sinh (\gamma \mathcal{T}) \sinh (\gamma \mathcal{U})}{\sinh (\gamma(\mathcal{S}+\mathcal{T})) \sinh (\gamma(\mathcal{T}+\mathcal{U})) \sinh (\gamma(\mathcal{U}+\mathcal{S}))}\right)^{2}$.

Note that the continuous three-point function is now

$$
\begin{aligned}
& \tilde{\mathcal{G}}\left(\mathcal{D}_{12}, \mathcal{D}_{13}, \mathcal{D}_{23}\right)=\frac{1}{2} \partial_{\mathcal{S}} \partial_{\mathcal{T}} \partial_{\mathcal{U}} \tilde{\mathcal{F}}(\mathcal{S}, \mathcal{T}, \mathcal{U}) \\
& \text { where } \mathcal{D}_{12}=\mathcal{S}+\mathcal{T}, \mathcal{D}_{13}=\mathcal{S}+\mathcal{U}, \mathcal{D}_{23}=\mathcal{T}+\mathcal{U},
\end{aligned}
$$

with a $1 / 2$ factor arising from the parity constraint on the sum of the three pairwise distances. Again, the (normalized) continuous canonical three-point function is exactly the same as that 
for quadrangulations or general maps except for the global change of scale of the renormalized distances through the scaling factor $\gamma$ of 100 .

\section{Conclusion}

In this paper, we established several bijections between bi-pointed or tri-pointed, general or bipartite, planar maps with prescribed distances between their marked vertices and suitably defined well-labelled or very-well-labelled objects. From the generating functions of these latter objects, we were able to recover known formulas for the two-point function of maps and to derive new explicit expressions for their three-point function, with a control on both their numbers of edges and faces.

The techniques that we used are in all respects similar to those used in [8] where the same questions were addressed in the context of planar quadrangulations. The intermediate welllabelled building blocks are essentially the same, with only slight differences in the game rules: for instance, while the three-point function of quadrangulations involves $(s, t, u)^{+}$well-labelled maps enumerated by $X_{s, t} X_{s, u} X_{t, u} Y_{s, t, u}^{2}$, that of general maps is restricted to the subclass of those maps being of type A, enumerated by $N_{s, t} N_{s, u} N_{t, u} Y_{s, t, u}^{2}$ or of type B, enumerated by $O_{s, t} O_{s, u} O_{t, u} Y_{s, t, u}^{2}$.

These small differences, although crucial at the discrete level, turn out to disappear in the scaling limit of large maps. In particular, the continuous canonical two- and three-point functions of general planar maps with a large number $n$ of edges are exactly the same as those of planar quadrangulations with a large number $n$ of faces. As a consequence, all the different limiting situations discussed in 8 are recovered for general maps.

As for general or bipartite planar maps enumerated with a fixed value of the weight $z$ per face, their continuous canonical two- and three-point functions at large number $n$ of edges are again the same as for quadrangulations up to a global, $z$-dependent, change of scale in the distances by the scaling factor $\gamma$ of $(84)$ or 100 . In this sense, these functions are universal and this corroborates the belief that all the families of maps considered here lie in the same universality class, that of so-called "pure gravity". In another, more probabilistic direction, this universality is also confirmed, for $z=1$, by the fact that the so-called Brownian map 13 is the universal scaling limit of general and bipartite maps counted by their number of edges [4, 1].

Note that the universality class of pure gravity is reached provided $\gamma$ is finite and nonzero, which therefore excludes the cases $z \rightarrow 0(\gamma \rightarrow 0)$ and $z \rightarrow \infty(\gamma \rightarrow \infty)$, which are thus in a different universality class. For $z \rightarrow 0$, one finds (omitting prefactors) that, for both general and bipartite maps, $\gamma \simeq z^{1 / 6}$ and $n_{f} / n \simeq z^{2 / 3}$, and therefore $\gamma \simeq\left(n_{f} / n\right)^{1 / 4}$. This suggests that, when dealing with an ensemble where we impose that $n_{f} \simeq n^{b}$ for some $b$, with $0 \leq b<1$, the statistics of distances should be describable by an effective $n$-dependent scaling factor $\gamma$ satisfying $\gamma \simeq n^{(b-1) / 4}$. This would indicate that the typical (discrete) distance $d$, of order $n^{1 / 4} / \gamma$, would scale as $d \simeq n^{(2-b) / 4}$. For $b=0$ (i.e., with a finite number of faces), this gives $d \simeq n^{1 / 2}$ as expected. For $b \rightarrow 1$, the exponent tends to $1 / 4$ as expected. For $z \rightarrow \infty$ instead, one finds for general maps $\gamma \simeq z^{1 / 6}$ and $n_{v} / n \simeq z^{-2 / 3}$, and for bipartite maps $\gamma \simeq z^{1 / 8}$ and $n_{v} / n \simeq z^{-1 / 2}$. Therefore $\gamma \simeq\left(n_{v} / n\right)^{-1 / 4}$ in both cases. Hence, in an ensemble where we impose that $n_{v} \simeq n^{c}$ for some $c$, with $0 \leq c<1$, we expect that the statistics of distances should be governed by an effective $n$-dependent scaling factor $\gamma \simeq n^{(1-c) / 4}$. This would indicate that the typical (discrete) distance $d$, of order $n^{1 / 4} / \gamma$, would now scale as $d \simeq n^{c / 4}$. For $c=0$ (i.e., with a finite number of vertices), this gives $d \simeq n^{0}$ as expected, since distances remain finite in maps with a finite number of vertices. For $c \rightarrow 1$, the exponent tends to $1 / 4$ as expected. All these conjectures require careful calculations to be confirmed.

Even in the scaling limit, some non-universal quantities (other than the scaling factor) can be read off the normalizations of the various scaling functions: we discussed for instance the asymptotic average numbers of vertices and faces in maps with a fixed weight $z$ per face, 
as well as the number of geodesic vertices and edges between two points far away. Many other non-universal quantities follow from our formulas: in particular, we could also easily explore the (non-universal) so-called local limit, corresponding to vertices at a finite discrete distance in large (potentially infinite) maps.

To conclude, let us mention that, in the context of quadrangulations, several refinements of [8] led to a more precise description of the "geodesic triangle" formed by three random points (with in particular a measure of the so-called "confluence phenomenon" for geodesics) as well as to an evaluation of the distance properties of "separating loops" 9]. There should not be any difficulty to apply the same refinements to our calculations and address similar questions now in the context of general or bipartite planar maps.

\section{ACKNOWLEDGEMENTS}

The work of ÉF was partly supported by the ANR grant "Cartaplus" 12-JS02-001-01 and the ANR grant "EGOS" 12-JS02-002-01.

\section{REFERENCES}

[1] C. Abraham. Rescaled bipartite planar maps converge to the Brownian map, 2014. In preparation.

[2] J. Ambjørn and T.G. Budd. Trees and spatial topology change in causal dynamical triangulations. J. Phys. A: Math. Theor., 46(31):315201, 2013.

[3] J. Ambjørn and Y. Watabiki. Scaling in quantum gravity. Nuclear Phys. B, 445(1):129-142, 1995.

[4] J. Bettinelli, E. Jacob, and G. Miermont. The scaling limit of uniform random plane maps, via the Ambjørn-Budd bijection, 2013. arXiv:1312.5842 [math.PR].

[5] J. Bouttier, P. Di Francesco, and E. Guitter. Geodesic distance in planar graphs. Nucl. Phys. B, $663(3): 535-567,2003$.

[6] J. Bouttier, P. Di Francesco, and E. Guitter. Planar maps as labeled mobiles. Electron. J. Combin., 11(1):R69, 2004.

[7] J. Bouttier, É. Fusy, and E. Guitter. On the two-point function of general planar maps and hypermaps, 2013. arXiv:1312.0502 [math.CO].

[8] J. Bouttier and E. Guitter. The three-point function of planar quadrangulations. J. Stat. Mech., 2008(07):P07020, 2008.

[9] J. Bouttier and E. Guitter. Confluence of geodesic paths and separating loops in large planar quadrangulations. J. Stat. Mech., 2009(03):P03001, 2009.

[10] J. Bouttier and E. Guitter. Planar maps and continued fractions. Comm. Math. Phys., 309(3):623-662, 2012.

[11] G. Chapuy, M. Marcus, and G. Schaeffer. A bijection for rooted maps on orientable surfaces. SIAM J. Discrete Math., 23(3):1587-1611, 2009.

[12] R. Cori and B. Vauquelin. Planar maps are well labeled trees. Canad. J. Math., 33(5):1023-1042, 1981.

[13] J.-F. Le Gall. The topological structure of scaling limits of large planar maps. Inventiones mathematicae, 169(3):621-670, 2007.

[14] G. Miermont. Tessellations of random maps of arbitrary genus. Ann. Sci. Éc. Norm. Supér. (4), 42(5):725-781, 2009.

[15] G. Schaeffer. Conjugaison d'arbres et cartes combinatoires aléatoires. PhD thesis, Université Bordeaux I, 1998.

LiX, École Polytechnique, 91128 Palaiseau, France

E-mail address: fusy@lix.polytechnique.fr

Institut de Physique Théorique, CEA, IPht, 91191 Gif-sur-Yvette, France, CNRS, URA 2306

E-mail address: emmanuel.guitter@cea.fr 